\title{
Clathrin Assembly Protein AP180 and CALM Differentially Control Axogenesis and Dendrite Outgrowth in Embryonic Hippocampal Neurons
}

\author{
Ittai Bushlin, ${ }^{1}$ Ronald S. Petralia, ${ }^{2}$ Fangbai Wu, ${ }^{1}$ Asaff Harel, ${ }^{1}$ Mohamed R. Mughal, ${ }^{1}$ Mark P. Mattson, ${ }^{1}$ and \\ Pamela J. Yao ${ }^{1}$ \\ ${ }^{1}$ Laboratory of Neurosciences, National Institute on Aging-National Institutes of Health, Baltimore, Maryland 21224, and ${ }^{2}$ Laboratory of Neurochemistry, \\ National Institute on Deafness and Other Communication Disorders-National Institutes of Health, Bethesda, Maryland 20892
}

\begin{abstract}
Emerging data suggest that, much like epithelial cells, the polarized growth of neurons requires both the secretory and endocytic pathways. The clathrin assembly proteins AP180 and CALM (clathrin assembly lymphoid myeloid protein) are known to be involved in clathrin-mediated endocytosis, but their roles in mammalian neurons and, in particular, in developmental processes before synaptogenesis are unknown. Here we provide evidence that AP180 and CALM play critical roles in establishing the polarity and controlling the growth of axons and dendrites in embryonic hippocampal neurons. Knockdown of AP180 primarily impairs axonal development, whereas reducing CALM levels results in dendritic dystrophy. Conversely, neurons that overexpress AP180 or CALM generate multiple axons. Ultrastructural analysis shows that CALM affiliates with a wider range of intracellular trafficking organelles than does AP180. Functional analysis shows that endocytosis is reduced in both AP180-deficient and CALM-deficient neurons. Additionally, CALMdeficient neurons show disrupted secretory transport. Our data demonstrate previously unknown functions for AP180 and CALM in intracellular trafficking that are essential in the growth of neurons.
\end{abstract}

Key words: AP180; CALM; trafficking; neuron; polarity; development

\section{Introduction}

Proper intracellular trafficking is crucial for controlling the growth and establishing the polarity of many cells, including neurons. Studies have found that blocking forward Golgi transport impedes the growth of axons (Jareb and Banker, 1997) and dendrites (Horton et al., 2005) in developing hippocampal neurons. Similarly, Drosophila mutants lacking functional endoplasmic reticulum (ER)-to-Golgi trafficking display impaired dendritic growth (Ye et al., 2007). These experiments indicate that trafficking along secretory pathways dictates polarized growth of the neuron by directing the specifically required protein and lipid constituents to axons or dendrites.

Endocytosis also plays an important role in the distribution and abundance of polarized proteins including axonal accumulation of sodium channel Nav1.2 (Garrido et al., 2001) and synaptic vesicle protein VAMP2 (Sampo et al., 2003). In addition to targeting those proteins that directly contribute to neuronal

Received June 1, 2008; revised July 29, 2008; accepted Aug. 18, 2008.

This work was supported by the Intramural Research Program of the National Institutes of Health (NIH)-National Institute on Aging and $\mathrm{NIH}-$ National Institute on Deafness and Other Communication Disorders. We wish to thank Drs. Ernst J. Ungewickell for the AP180 CDNA, Jennifer Lippincott-Schwartz for the VSVG-GFP, Joshua R. Sanes for the VAMP2-BTX, Douglas S. Lyles for the VSVG antibody, and Peter Davies for the PHF-1 antibody. We also thank Dr. Ya-Xian Wang for help with specimen preparation.

Correspondence should be addressed to Dr. Pamela J. Yao, Laboratory of Neurosciences, The National Institute on Aging-National Institutes of Health Biomedical Research Center, 251 Bayview Boulevard, Baltimore, MD 21224. E-mail:yaopa@grc.nia.nih.gov.

DOI:10.1523/JNEUROSCI.2471-08.2008

Copyright $\odot 2008$ Society for Neuroscience $\quad$ 0270-6474/08/2810257-15\$15.00/0 function, endocytosis also seems involved in targeting proteins that control the process of polarization itself. A study of budding yeast finds that the endocytosis rate has a profound impact on the polarized distribution of $\mathrm{Cdc} 42$, which in turn determines its function in regulating cell polarity (Marco et al., 2007). Cdc42 is a member of the evolutionary conserved PAR3-PAR6-aPKCCdc42 complex, which is crucial for polarity establishment in many cell types (Goldstein and Macara, 2007), hippocampal neurons among them (Shi et al., 2003).

Clathrin-coated vesicles (CCVs) are best understood for their roles in endocytosis at the plasma membrane and transport from the trans-Golgi network (TGN) to endosomes at the late secretory pathway (Brodsky et al., 2001). The CCV is composed of the clathrin triskelion, the plasma membrane, and the heterotetramer adaptor protein (AP). The primary function of APs is to anchor the clathrin to the plasma membrane and to select some, but not all, cargo for inclusion into CCV (Owen et al., 2004; Robinson, 2004).

AP180 (Kohtz and Puszkin, 1988; Murphy et al., 1991; Morris et al., 1993; Zhou et al., 1993) and CALM (clathrin assembly lymphoid myeloid protein) (Dreyling et al., 1996; Tebar et al., 1999) belong to a group of proteins with clathrin assembly activity. Functional studies performed in in vitro systems or in invertebrates have indicated that AP180 and CALM perform similar roles: facilitating the assembly of CCVs and controlling the size of CCVs (Ahle and Ungewickell, 1986; Ye and Lafer, 1995; Zhang et al., 1998; Morgan et al., 1999; Meyerholz et al., 2005). AP180 is 
expressed only in neurons (Sousa et al., 1992; Yao et al., 2002, 2005), whereas CALM is expressed in cells of all tissues, including neurons (Dreyling et al., 1996; Yao et al., 2005; Petralia and Yao, 2007). In addition to mature neurons, both proteins are highly expressed in young developing neurons (Petralia and Yao, 2007). However, little is known about the physiological roles of AP180 and CALM in the mammalian neuron, and particularly about their role in the early events in the formation of neuronal circuits. Here we show that AP180 and CALM play pivotal roles in the clathrin-mediated trafficking pathways that control cell polarity and axon and dendrite outgrowth in embryonic hippocampal neurons.

\section{Materials and Methods}

DNA constructs. The AP180 tagged with hemagglutinin epitope (AP180HA) was generated by polymerase chain reaction using the rat AP180 cDNA (kindly provided by Dr. Ernst J. Ungewickell, Hannover Medical School, Hannover, Germany) (Morris et al., 1993). For the AP180ANTH-HA (AP180-folded N-terminal homology-hemagglutinin epitope) deletion mutant, the amplified DNA fragment (supplemental Fig. S7A, available at www.jneurosci.org as supplemental material) was ligated into the AflII and KpnI restriction sites of the expression vector pcDNA3.1; for the AP180-ANTH $\Delta$-HA, the amplified DNA was ligated into the EcoI and NotI sites of pcDNA3.1. For the AP180siRNA (small interfering RNAs), 5'-GATCCCCACAACGCCTTCGACTTCAATTCAAGAGATTGAAGTCGAAGGCGTTGTTTTTTC-3' and 5'-TCGAGAAAAAACAACGCCTTCGACTTCAATCTCTTGAATTGAAGTCGAAGGCGTTGTGGG-3' (corresponding to nucleotides 2157-2175 of rat AP180) were annealed and subcloned into the BglII and XhoI sites of the expression vector pSuper (OligoEngine). For the control AP180siRNA, $7 \mathrm{nt}$ within the targeting region were inverted (supplemental Fig. S1 A, available at www.jneurosci.org as supplemental material). The AP180siRNA-resistant AP180 containing silent point mutations (supplemental Fig. S1 A, available at www.jneurosci.org as supplemental material) was generated using the AP180-HA as a template and the QuickChange site-directed mutagenesis kit (Stratagene).

The wild-type CALM, the CALM deletion constructs, the CALMsiRNA, the control CALMsiRNA, and the CALMsiRNA-resistant CALM have been described previously (Harel et al., 2008). Mouse CALM long splice variant (CALM-L; GenBank accession number BC011470) and short splice variant (CALM-S; GenBank accession number BC021491) from ATCC were used as templates.

The VAMP2 construct containing a C-terminal $\alpha$-bungarotoxinbinding site tag (VAMP2-BTX) was a kind gift from Dr. Joshua R. Sanes (Harvard University, Cambridge, MA) (McCann et al., 2005). The VAMP2 containing a single amino acid mutation, M46A, was generated using the VAMP2-BTX as a template and the QuikChange site-directed mutagenesis kit. The VSVG-GFP [vesicular stomatitis virus glycoprotein (ts045) tagged with green fluorescent protein] was a kind gift from Dr. Jennifer Lippincott-Schwartz (National Institutes of Health, Bethesda, MD) (Presley et al., 1997). All constructs were verified by DNA sequencing.

Antibodies and other regents. The following antibodies were used: AP180 (clone AP180-I; Sigma), CALM (\#sc5395 and \#sc6433; Santa Cruz Biotechnology), clathrin (clone TD.1; Abcam), actin (clone AC-15; Sigma), Tuj1 (rabbit serum; Covance), taul (clone Tau-1; Millipore Bioscience Research Reagents), MAP2 (clone AP-20; Sigma), smi312 (clone SMI-312; Covance), PHF1 (kind gift from Dr. Peter Davies, Albert Einstein College of Medicine, Yeshiva University, New York, NY), HA (clone 16B12; Covance), and VSVG (clone 11; kind gift from Dr. Douglas S. Lyles, Wake Forest University, Winston-Salem, NC). The following antibodies were from BD Biosciences: EEA1, GM130, TGN38, and AP1 $\gamma$. Alexa Fluor 568 secondary antibodies and Alexa Fluor 555 $\alpha$-bungarotoxin used in fluorescent labeling were from Invitrogen. Peroxidase-conjugated secondary antibodies were from Jackson ImmunoResearch Laboratories. Gold-conjugated secondary antibodies were from Ted Pella.

Cell culture and transfection. Cultures of hippocampal neurons were prepared from embryonic day 18 rat brains as described previously (Kaech and Banker, 2006). Dissociated neurons $\left(\sim 150\right.$ cells $\left./ \mathrm{mm}^{2}\right)$ were plated on coverslips coated with poly-D-lysine and grown in Neurobasal medium (Invitrogen) supplemented with B27 (Invitrogen) and $0.5 \mathrm{~mm}$ glutamine. Neurons were transfected using the calcium phosphate kit (Invitrogen) and analyzed $4 \mathrm{~d}$ after transfection unless otherwise indicated. The transfection efficiency of the cultured hippocampal neurons was 0.5 to $1 \%$. For cotransfection with enhanced green fluorescent protein (EGFP), pEGFP was mixed with the testing plasmid at the ratio of 1:2. For the siRNA rescue experiment, the siRNA plasmid was mixed with the siRNA-resistant plasmid in the ratio of 1:1. Human embryonic kidney (HEK) 293 cells obtained from ATCC were cultured according to the manufacturer's instructions and transfected using the calcium phosphate kit.

Immunocytochemistry, fluorescence microscopy, and image analysis. For intracellular labeling, transfected neurons were fixed with $4 \%$ paraformaldehyde and $4 \%$ sucrose for $15 \mathrm{~min}$, washed, permeabilized in PBS containing $0.1 \%$ Triton X-100, and blocked in 10\% BSA or $10 \%$ appropriate normal serum. Neurons were incubated with a primary antibody overnight at $4^{\circ} \mathrm{C}$. The neurons were washed and incubated with an appropriate fluorescently tagged secondary antibody. For surface labeling, neurons were fixed and labeled without permeabilization.

The labeled neurons were examined and images were captured using a $40 \times$ or a $63 \times$ objective on a Zeiss LSM510 laser scanning confocal microscope. All images were acquired at a $1024 \times 1024$ pixel resolution and each image was an average of four scans at the same focal plane. The confocal acquisition settings were kept the same for those samples when fluorescence intensities were compared.

Two or three coverslips from each culture were used for analysis. In a typical case, there were 10-20 transfected neurons in a coverslip. In each coverslip, we randomly selected the first 10 transfected neurons regardless of the morphology and photographed them using the criteria described above. Photos were scored twice by two observers (A. H. and F. W.) who were unaware of the experimental details until after the analysis was complete.

The length and the number of neurites were measured from EGFP images of transfected neurons using NeuronJ. To measure axons or dendrites, both the EGFP image and the tau1 or MAP2 image of each neuron was used to distinguish the transfected neuron from neighboring untransfected neurons, and to identify the axon or dendrite. For Sholl analysis, concentric circles with $10 \mu \mathrm{m}$ differences in diameter were drawn around the soma, and the number of neurites crossing each circle was quantified using ImageJ (Sholl analysis Plugin). For quantifying the AP180-HA or the CALM-HA deletion mutants in neurites, a region of interest was drawn on a neurite and a soma, the average intensity of HA labeling was determined using ImageJ, and the neurite/soma ratio was obtained. For quantifying taul enrichment, the average fluorescence intensity of taul labeling in the longest neurite was divided by that in the soma.

Analysis of VSVG trafficking. VSVG-GFP was cotransfected with the siRNA at the ratio of $1: 1$. After incubation at $40^{\circ} \mathrm{C}$ overnight, the transfected neurons were shifted to $32^{\circ} \mathrm{C}$ incubation for $2 \mathrm{~h}$. The neurons were fixed without permeabilization and labeled using the VSVG antibody (Lefrancios and Lyles, 1982). The average intensity of surface VSVG detected by the antibody and of total VSVG-GFP was determined using ImageJ and the surface/total VSVG ratio was obtained.

Analysis of surface VAMP2-BTX labeling. To label surface VAMP2BTX, live transfected neurons were incubated with Alexa Fluor 555conjugated BTX $(2 \mu \mathrm{g} / \mathrm{ml})$ in $\mathrm{Ca}^{2+}$-free saline at $4^{\circ} \mathrm{C}$ for $30 \mathrm{~min}$. The neurons were washed in cold fluorophore-free and $\mathrm{Ca}^{2+}$-free saline and fixed. To quantify the axonal enrichment of surface VAMP2-BTX, the average intensity of BTX florescence in the longest neurite was divided by that in the soma and all other neurites combined.

Electron microscopy. Postembedding, processed hippocampal sections from postnatal day 10 (P10) and P37 rats were the same materials obtained from previous studies (Petralia et al., 2005; Yao et al., 2005; Petralia and Yao, 2007). Immunogold labeling was performed exactly as described previously (Petralia and Wenthold, 1999; Petralia and Yao, 2007). Sections were incubated with the AP180 antibody (1:25) or the 
Table 1. Ultrastructural localization of AP180 and CALM in relation to the plasma membrane

\begin{tabular}{lllll}
\hline & & $\leq 100 \mathrm{~nm}$ & $>100 \mathrm{~nm}$ & Total gold \\
\hline \multirow{2}{*}{ AP180 } & P10 & $65 \%$ & $35 \%$ & 395 \\
& Adult & $71 \%$ & $29 \%$ & 300 \\
\multirow{2}{*}{ ALM } & P10 & $55 \%$ & $45 \%$ & 969 \\
& Adult & $58 \%$ & $42 \%$ & 988 \\
\hline
\end{tabular}

CALM antibody (1:50 for both \#sc5395 and \#sc6433) at $4^{\circ} \mathrm{C}$ overnight. After thorough washing, sections were incubated with appropriate gold $(10 \mathrm{~nm})$-conjugated antibodies. The sections were contrasted with uranyl acetate and lead citrate and examined on the electron microscope at a magnification of $25,000 \times$ or $50,000 \times$

For quantitative analysis of subcellular distribution (see Fig. 6A, Table 1 ), we focused on the CA1 stratum radiatum of the hippocampus. In each section, we examined all of the grid hexagons and systematically photographed all of the fields within each grid. Immunolabeling was considered positive if at least two gold particles were present within a clearly identifiable plasma membrane.

For analysis of intracellular CALM labeling in relation to intracellular organelles, we examined the CA1 stratum pyramidale and stratum radiatum as well as the CA3 stratum lucidum with a focus on large dendrites and the cell body. For analysis of CALM localization in relation to dendritic branch points, we examined the CA1 stratum radiatum and stratum oriens.

All images were stored in their original formats. Images for printing were prepared with Adobe Photoshop. Brightness/contrast or levels of images were minimally adjusted. No further manipulations were used. The Photoshop-processed image layouts were imported into Adobe Illustrator and the labels were added.

Data analysis and statistics. Statistical analysis was performed with the $t$ test or ANOVA. All results were expressed as mean \pm SEM.

\section{Results}

Reduction of AP180 and CALM causes different growth defects in hippocampal neurons

AP180 and CALM are highly expressed in embryonic hippocampal neurons in vitro (Yao et al., 2003) and in young hippocampal neurons in vivo (Petralia and Yao, 2007). To investigate the function of AP180 and CALM in developing neurons, we used siRNAs to knock down AP180 or CALM in cultured embryonic hippocampal neurons. A DNA plasmid (pSuper) (Brummelkamp et al., 2002) containing 19 nt sequences specific to AP180 (pSuperAP180siRNA) (supplemental Fig. S1A, available at www. jneurosci.org as supplemental material) or CALM (pSuperCALMsiRNA) (Harel et al., 2008) was generated. We validated the specificity and efficacy of AP180siRNA in knocking down AP180 protein expression by immunoblotting of HEK293 cells (supplemental Fig. S1 B, available at www.jneurosci.org as supplemental material) and immunolabeling of hippocampal neurons (supplemental Fig. S1C, available at www.jneurosci.org as supplemental material). The reduction of CALM protein levels in non-neuronal cells by CALMsiRNA has been described in a previous study (Harel et al., 2008). In the hippocampal neurons as assessed by CALM immunolabeling (supplemental Fig. S2, available at www.jneurosci.org as supplemental material) and intensity measurement of the immunolabeling, CALMsiRNA reduced CALM protein levels by $\sim 69 \%$ (70 $\pm 5.1 \%, n=45$ neurons). Using the propidium iodide exclusion assay, we did not observe altered cell viability in either AP180siRNA- or CALMsiRNAtransfected neurons (data not shown).

We transfected cultured hippocampal neurons at 2 days in vitro (div) with either AP180siRNA or CALMsiRNA, together with EGFP to mark transfected neurons and to visualize their morphology. We examined neuronal morphology $4 \mathrm{~d}$ after transfection $(2+4=6 \mathrm{div})$. The growth pattern of dissociated hippocampal neurons in culture has been well characterized (Kaech and Banker, 2006). In keeping with this characterization, we found in our system that a typical neuron between 2 and 6 div, without transfection, developed an average of five to six neurites. One of the neurites was readily distinguishable: it was at least 2-3 times longer than other neurites and was immunolabeled for the axonal marker tau1 (supplemental Fig. S3, available at www. jneurosci.org as supplemental material). Neurons cotransfected with the empty vector pSuper and EGFP were used as the control, because these control neurons showed a morphology (Fig. 1 A, vector) indistinguishable from nontransfected cells (supplemental Fig. S3, available at www.jneurosci.org as supplemental material).

Neurons expressing AP180siRNA had five to six neurites. Many of these neurons, however, no longer possessed a single long axon-like neurite, resulting in a loss of the characteristic neuronal polarity (Fig. 1A; supplemental Fig. S4A, available at www.jneurosci.org as supplemental material). Although the number of neurites was not significantly changed (Fig. $1 B$ ), measurements of total neurite length on AP180siRNA neurons revealed an $\sim 20 \%$ reduction $(579 \pm 47 \mu \mathrm{m}$ vs $726 \pm 48 \mu \mathrm{m}, p<$ $0.05, n=60$ ) (Fig. 1C). Neurons expressing CALMsiRNA also exhibited a morphological change, but this change was distinctively different from the AP180siRNA-induced phenotype. Instead of the typical five to six neurites, many CALMsiRNA neurons were left with only one long neurite (Fig. $1 A$; supplemental Fig. S4 A, available at www.jneurosci.org as supplemental material). Not surprisingly, the number of neurites was significantly reduced in these cells (Fig. $1 B$ ), and the total neurite length showed an $\sim 60 \%$ reduction ( $298 \pm 27 \mu \mathrm{m}$ vs $726 \pm 48 \mu \mathrm{m}, p<$ 0.001, $n=60$ ) (Fig. 1C).

To identify the type of neurites selectively affected by AP180siRNA or CALMsiRNA, we immunolabeled neurons for the axonal marker taul. At least $80 \%$ of 6 div neurons had one taul-labeled axon, whereas the remainder of the neurons either had no axon or had more than one axon (one axon, $80 \pm 7 \%$; no axon, $12 \pm 3 \%$; multiple axons, $8 \pm 4 \% ; n=60$ ) (Fig. $1 D, E$, vector; supplemental Fig. $S 4 B$, available at www.jneurosci.org as supplemental material). In neurons expressing AP180siRNA, however, we observed that the percentage of cells that did not display detectable taul labeling in any neurites increased to $\sim 60 \%(59 \pm 10 \%, n=60)$ (Fig. $1 E$; supplemental Fig. S4 $B$, available at www.jneurosci.org as supplemental material). Within this group of neurons, measurement of the average taul immunofluorescence intensity revealed that the ratio of tau 1 in neurites to that in the soma was lower than the ratio in the control neurons $(0.72 \pm 0.06$ vs $1.57 \pm 0.1, p<0.001)$ (supplemental Fig. $\mathrm{S} 4 \mathrm{C}$, available at www.jneurosci.org as supplemental material). Within the population of AP180siRNA neurons that still possessed tau1-positive axons, the length of the axons was significantly shorter than was observed in the control neurons (234 \pm $63 \mu \mathrm{m}$ vs $476 \pm 37 \mu \mathrm{m}, p<0.001 ; n=20$ for AP180siRNA, $n=$ 45 for control) (Fig. $1 F$ ). In neurons expressing CALMsiRNA, unlike neurons expressing AP180siRNA, we found readily detectable taul labeling in the longest neurite, the only neurite remaining in many such cells (Fig. 1D). Quantitative analysis revealed that whereas the proportion of cells containing one axon was unchanged ( $85 \pm 3 \%$ vs $80 \pm 7 \%$ in control neurons) (Fig. $1 E$; supplemental Fig. $S 4 B$, available at www.jneurosci.org as supplemental material), the length of axons was markedly reduced $(220 \pm 18 \mu \mathrm{m}$ vs $476 \pm 37 \mu \mathrm{m}, p<0.001, n=45)$ (Fig. $1 F)$. 


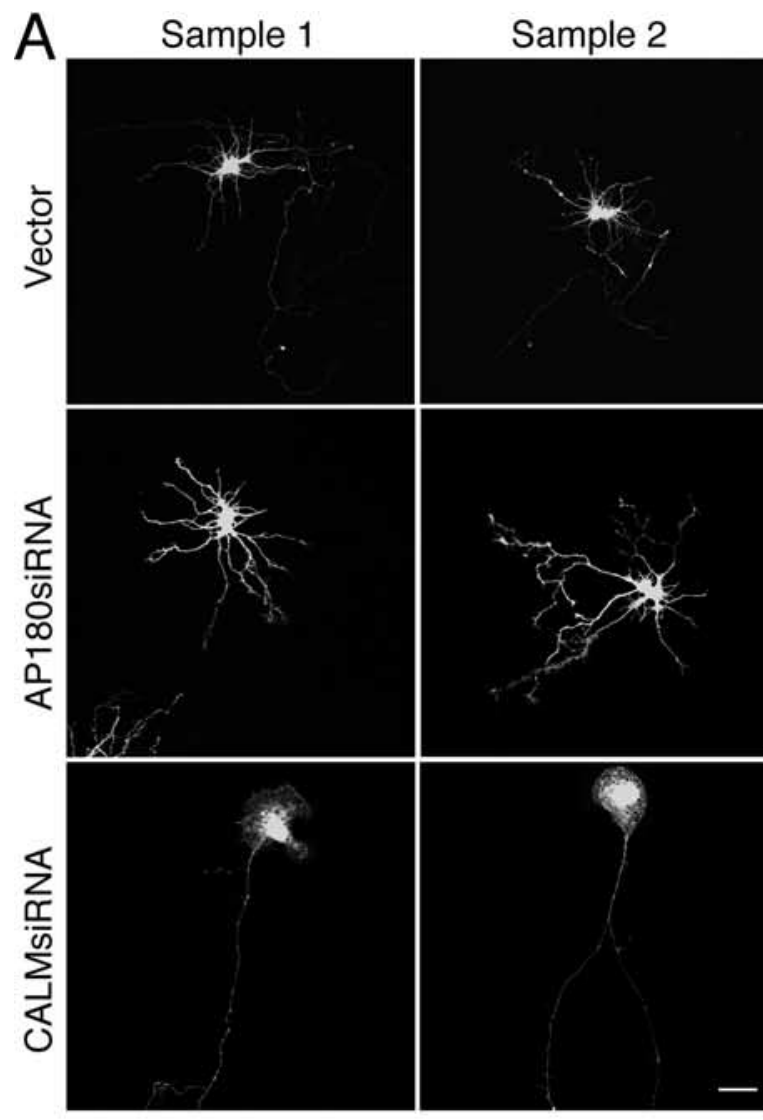

B

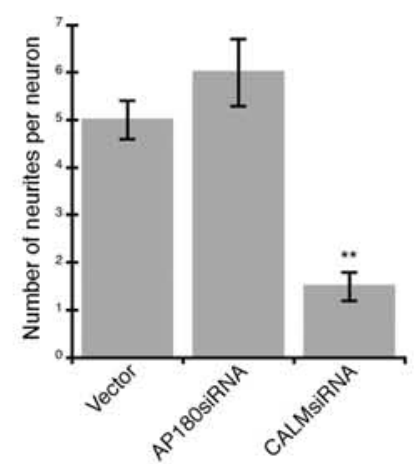

$\mathrm{E}$

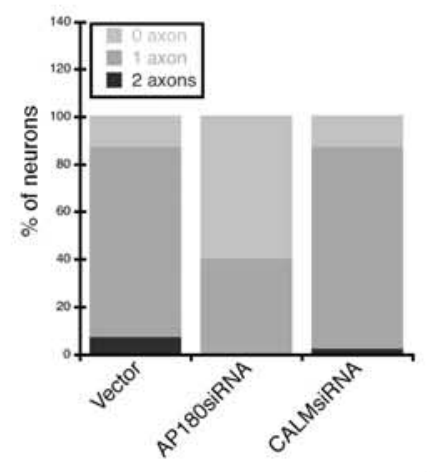

G

$\mathrm{D}$

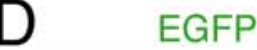

tau1

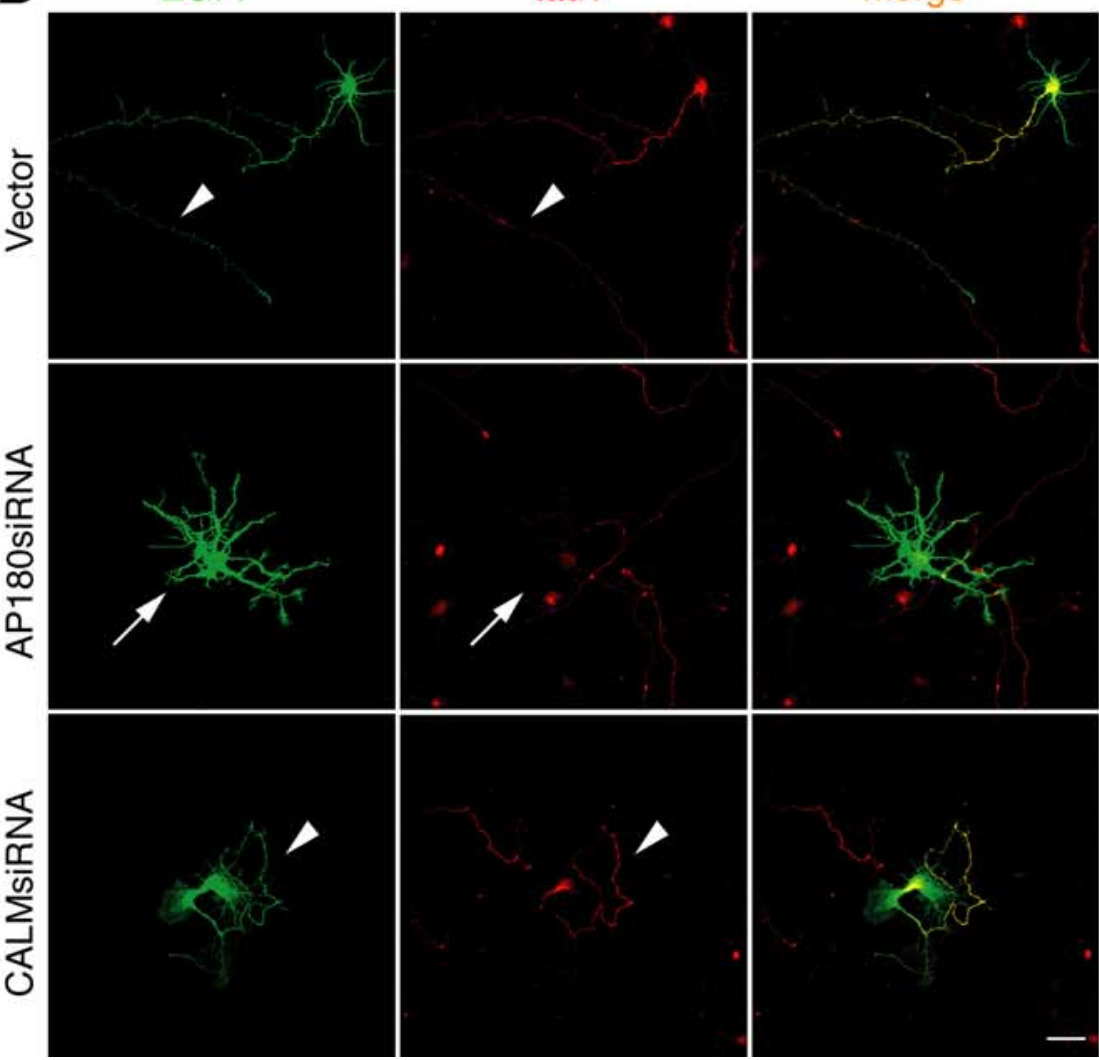

C

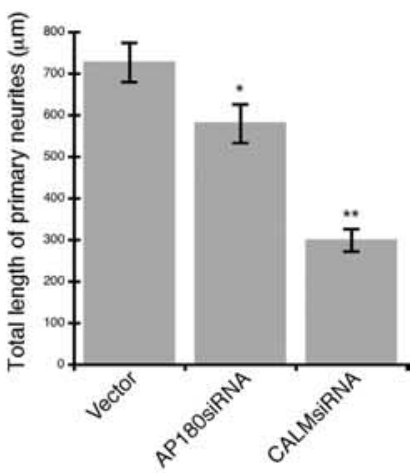

F
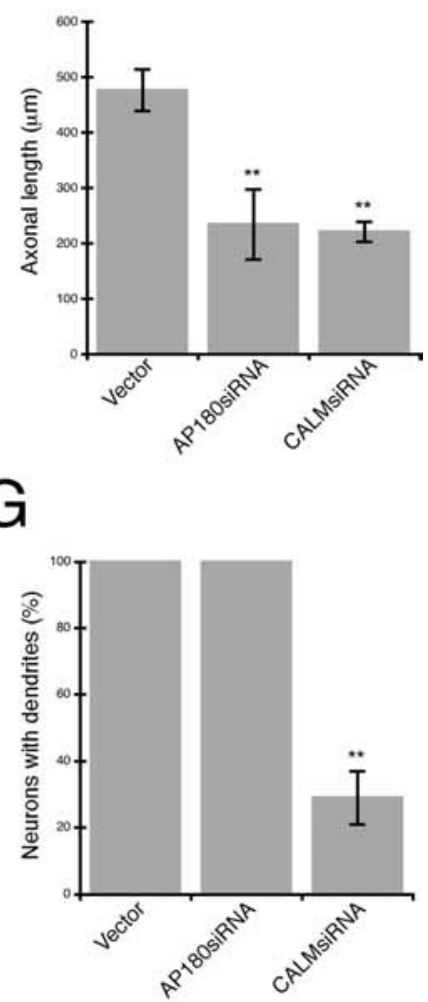

$\mathrm{H}$

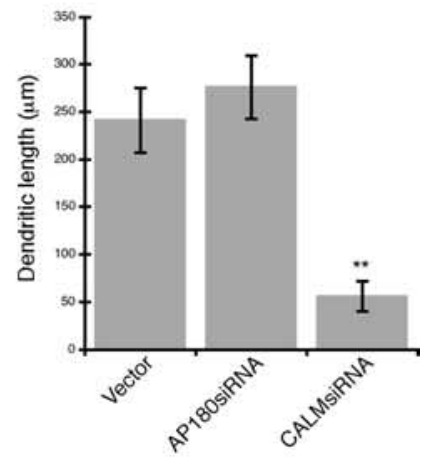

Figure 1. $\quad \boldsymbol{A}-\boldsymbol{H}$, Reduction of AP180 and CALM leads to different growth defects in hippocampal neurons. $\boldsymbol{A}$, Confocal images of representative embryonic hippocampal neurons expressing AP180siRNA or CALMsiRNA. Scale bar, $10 \mu \mathrm{m}$. Additional examples are shown in supplemental Figure $\$ 4 A$, available at www.jneurosci.org as supplemental material. $\boldsymbol{B}$, The number of neurites per neuron is not significantly changed in neurons expressing AP180siRNA but is reduced in neurons expressing CALMsiRNA. $n=60$ neurons in each group; ${ }^{* *} p<0.001$. $C$, Total neurite length is reduced in neurons expressing AP180siRNA and CALMsiRNA. $n=60$ neurons in each group; ${ }^{*} p<0.05,{ }^{* *} p<0.001$. D, Immunolabeling of the axonal (Figure legend continues.) 
We also immunolabeled neurons for the dendritic marker MAP2 (supplemental Fig. S4D, available at www.jneurosci.org as supplemental material). Nearly all neurons at 6 div had dendrites (Fig. 1G), and AP180siRNA neurons did not exhibit a measurable change in either the total length or number of primary dendrites $(n=45)$ (Fig. $1 H$; supplemental Fig. S4E, available at www. jneurosci.org as supplemental material). In contrast, the percentage of CALMsiRNA neurons with MAP2-labeled dendrites was reduced to $\sim 30 \%(29 \pm 8 \%, n=45)$ (Fig. $1 G)$. Within this group of cells, we sometimes found 1-2 MAP2 dendrites (supplemental Fig. S4E, available at www.jneurosci.org as supplemental material), and they were always significantly shorter than the dendrites in the control neurons ( $56 \pm 16 \mu \mathrm{m}$ vs $241 \pm 34 \mu \mathrm{m}, n=12, p<$ 0.001) (Fig. $1 H$ ).

To demonstrate the phenotypic specificity of the siRNAinduced effects, we next performed a rescue experiment by using a plasmid construct that contained an siRNA-resistant AP180 (supplemental Fig. S1 A, B, available at www.jneurosci.org as supplemental material) or a siRNA-resistant CALM (Harel et al., 2008). In neurons coexpressing the AP180siRNA and the siRNAresistant AP180, we focused on examining the axonal phenotype (Fig. 2A). A majority of these neurons had a proper axon (no rescue, $38 \pm 9 \%$ vs rescue, $79 \pm 8 \%, p<0.001, n=45)($ Fig. $2 B$ ) and normal axonal length (no rescue, $186 \pm 32 \mu \mathrm{m}$ vs rescue, $321 \pm 27 \mu \mathrm{m}, p<0.001$ ) (Fig. $2 C$ ). In neurons coexpressing the CALMsiRNA and siRNA-resistant CALM, we focused on the dendritic phenotype (Fig. 2D). We found that nearly all of the rescued cells had dendrites $(n=45)$ (Fig. $2 E)$ and that the dendritic length was significantly improved (no rescue, $59 \pm 18$ $\mu \mathrm{m}$ vs rescue, $161 \pm 27 \mu \mathrm{m}, p<0.01$; control, $n=40$; no rescue, $n=15$; rescue, $n=30$ ) (Fig. $2 F$ ). These results confirmed that the AP180siRNA- or CALMsiRNA-induced phenotypes were a consequence of the specific knockdown of either AP180 or CALM protein.

To follow the progress of the AP180 knockdown- or CALM knockdown-induced effects, we examined neurons at several time points by fixing cells from $1 \mathrm{~d}(2+1=3 \mathrm{div})$ to $4 \mathrm{~d}(2+4=$ 6 div) after siRNA transfection. During this period, the control neurons grew rapidly, with their outgrowth of axons increasing $\sim 4$-fold and dendrites increasing $\sim 2.5$-fold (Fig. $3 A, B$ ). In AP180 knockdown neurons, we began to observe a reduced axonal outgrowth at $2 \mathrm{~d}$ post-transfection, and the growth stopped at subsequent time points (Fig. $3 A$ ). Assessed by length only, AP180 knockdown did not appear to affect the total outgrowth of primary dendrites (Fig. 3B). In CALM knockdown neurons, we observed reduced axonal outgrowth in a pattern similar to the one observed in the AP180 knockdown neurons (Fig. 3A). The effect on dendritic growth, however, occurred earlier and was more severe. Within $1 \mathrm{~d}$ after CALMsiRNA transfection, dendritic growth had ceased and, in some cases, the neurons were completely devoid of dendrites (Fig. 3B).

Finally, we examined the effects of AP180 or CALM knock-

\footnotetext{
(Figure legend continued.) marker tau1 (red) in neurons coexpressing EGFP (green) and the indicated siRNA. Arrowheads indicate the tau1-labeled axon. Arrows mark an AP180siRNA neuron in which the axon is absent. Scale bar, $10 \mu \mathrm{m}$. $E$, Proportion of neurons containing tau1-labeled axons as shown in $\boldsymbol{D}$. $n=60$ neurons in each group. $\boldsymbol{F}$, The mean length of axons is reduced by both AP180siRNA and CALMsiRNA. Vector, $n=45$ neurons; AP180siRNA, $n=20$ neurons; CALMsiRNA $n=45$ neurons; ${ }^{* *} p<0.001$. G, Quantification of neurons containing MAP2-labeled dendrites. $n=45$ neurons in each group; ${ }^{* *} p<0.001$. $\boldsymbol{H}$, The total length of dendrites is reduced in the CALMsiRNA-expressing neurons. Vector and AP180siRNA, $n=45$; CALMsiRNA, $n=12 ;{ }^{* *} p<0.001$. Data represent means \pm SEM in $\boldsymbol{B}, \boldsymbol{C}, \boldsymbol{F}-\boldsymbol{H}$.
}

down in more mature neurons. We transfected neurons at 6 div and examined them at 10 div. Neurons at 10 div allowed us to examine more developed dendrites; examining axons, however, was no longer feasible because of their extensive length and interactions with neurites of other cells. In addition to tau1, we immunolabeled transfected neurons with two additional axonal markers: smi-312, a pan-axonal neurofilament marker (Sternberger et al., 1982); and PHF-1, an axonal marker for mature neurons. We found that labeled axons from transfected neurons intensely intertwined with axons, also labeled, from neighboring nontransfected neurons (supplemental Fig S5A, available at www.jneurosci.org as supplemental material). This precluded our accurately tracing of individual axons and differentiating between axons from various sources. Thus, we focused on examining dendrites.

The control neurons at this stage developed dendrites that were longer and more elaborate (Fig. 3C; supplemental Fig. S5B, available at www.jneurosci.org as supplemental material). Although the number of the primary dendrites in the AP180siRNA neurons appeared to be normal (supplemental Fig. S5C, available at www.jneurosci.org as supplemental material), these dendrites were noticeably simpler than those found in the control neurons (Fig. 3C; supplemental Fig. S5B, available at www.jneurosci.org as supplemental material). Sholl analysis revealed a significant reduction in dendritic complexity in the AP180 knockdown neurons (Fig. 3D). CALM knockdown neurons displayed the prototypical morphology (Fig. 3C; supplemental Fig. S5B, available at www.jneurosci.org as supplemental material), reminiscent of what we observed in younger neurons (Fig. $1 \mathrm{~A}$; supplemental Fig. S4, available at www.jneurosci.org as supplemental material). Nearly $90 \%$ of CALM knockdown neurons had only the distinctive single long neurite (Fig. $3 C$; Fig. $S 5 B-D$, available at www. jneurosci.org as supplemental material). Consistently, Sholl analysis revealed a marked reduction in both dendrite length and the overall complexity of the neurites (Fig. 3D). Together, these results suggest that AP180 and CALM have selective roles in neuronal growth, with AP180 being critical for axogenesis and CALM playing a major role in dendrite outgrowth.

\section{Overexpression of AP180 and CALM induces the formation of multiple axons}

Having observed that AP180 and CALM knockdown impair the outgrowth of, respectively, axons and dendrites, we next investigated whether overexpression of AP180 or CALM would induce any growth defect. Neurons were cotransfected with EGFP and AP180-HA or CALM-HA. For CALM, we examined the long (L) and short (S) splice variants. More than $90 \%$ of transfected cells coexpressed EGFP and AP180-HA or CALM-HA (data not shown).

The effects of AP180 and CALM overexpression were relatively subtle compared with the effects of depletion of endogenous AP180 and CALM. Neither AP180 nor CALM overexpression resulted in a noticeable change in total neurite outgrowth, assessed by neurite number and length (supplemental Fig. $\mathrm{S} 6 A, B$, available at www.jneurosci.org as supplemental material). However, we noted that, among neurons overexpressing AP180, the population of cells with multiple axons increased to $\sim 30 \%$ (31 $\pm 4 \%$ vs $9 \pm 2 \%, n=45$ ) Fig. $4 A, B$; supplemental Fig. $\mathrm{S} 6 C, D$, available at www.jneurosci.org as supplemental material). Similarly, neurons overexpressing CALM, CALM-L or CALM-S splice variant, also showed a moderate increase in the population with multiple axons (CALM-L, $20 \pm 3 \%$; CALM-S, $20 \pm 2 \%$; $n=$ 45) (Fig. $4 A, B$; supplemental Fig. S6C,D, available at www. 
A
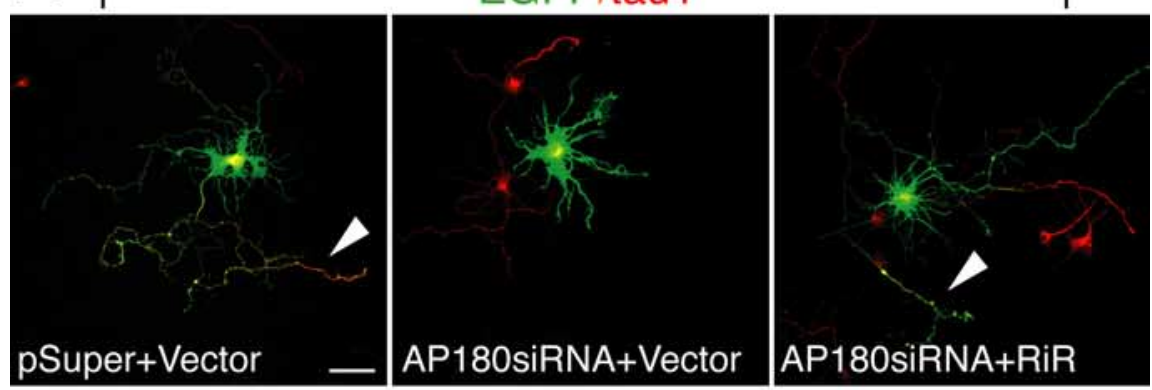

B

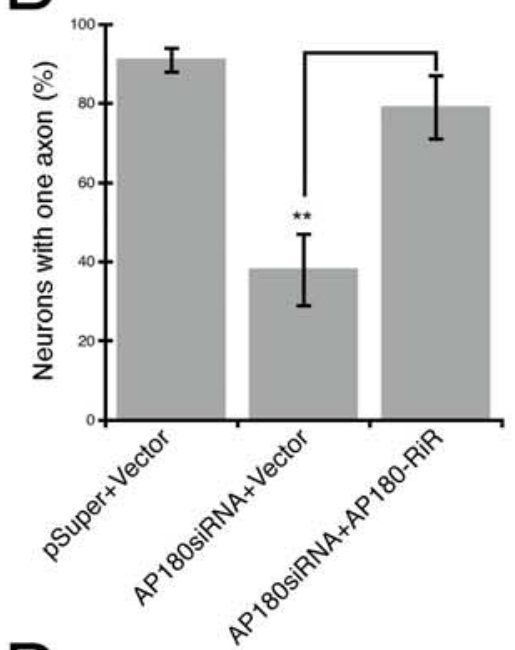

C

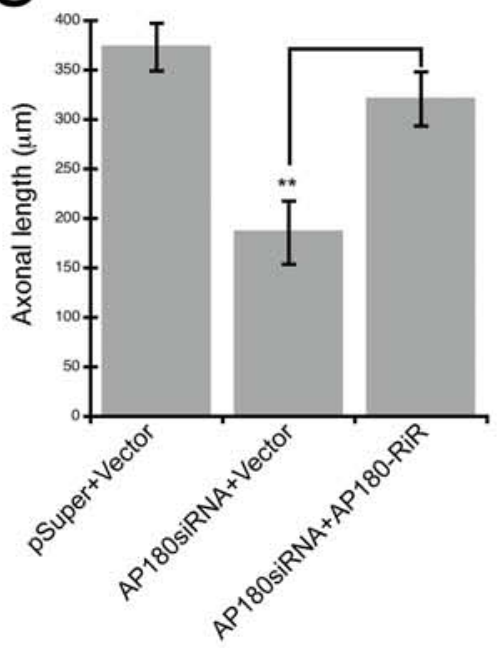

\section{EGFP/MAP2}
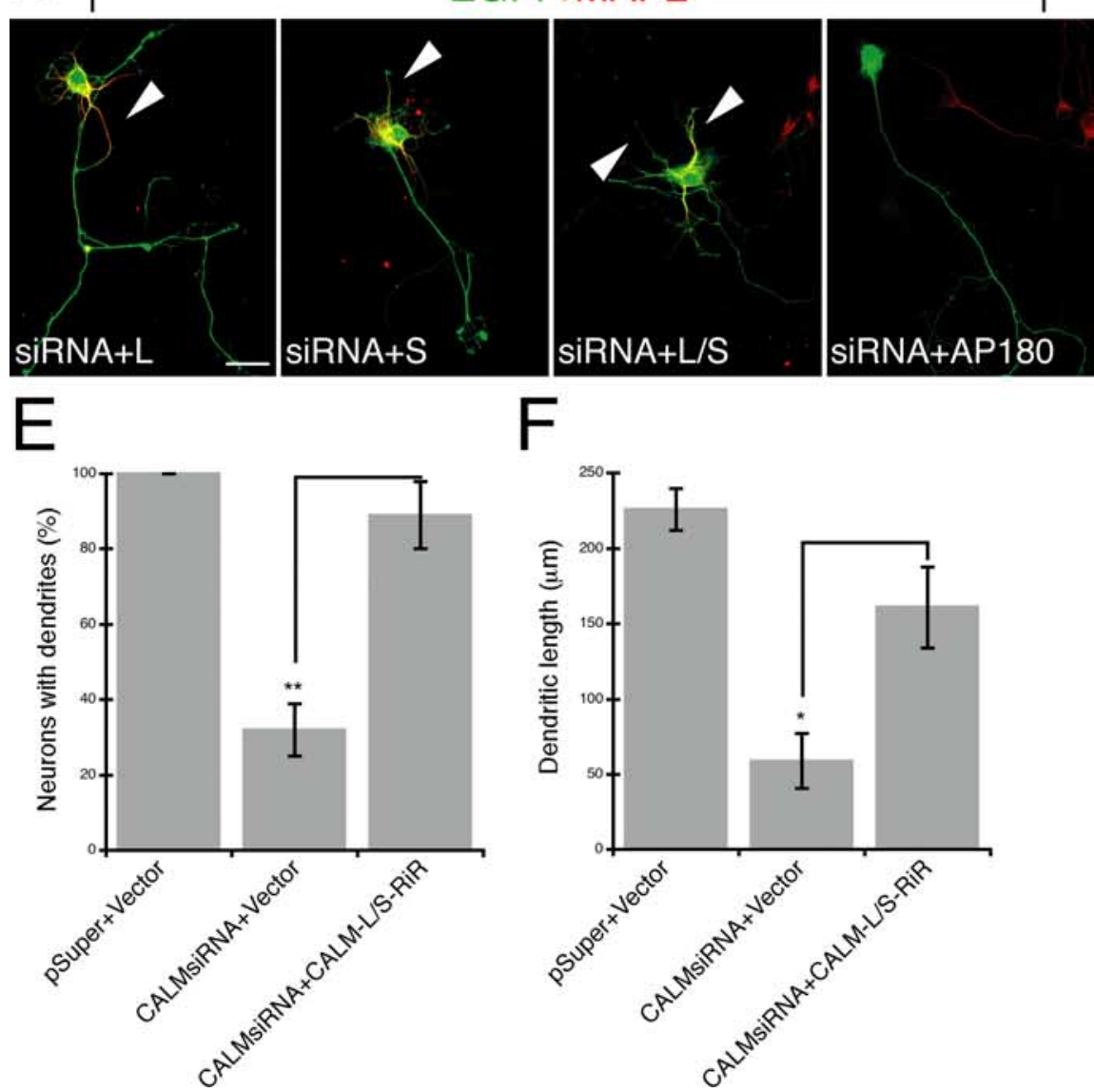

jneurosci.org as supplemental material). The axonal length of these neurons, however, did not appear to be significantly different from that of the control neurons (Fig. 4C). By immunolabeling with an HAspecific antibody, we confirmed that the axonal phenotype was associated with the ectopic expression of AP180-HA or CALM-HA in these neurons (supplemental Fig. S6E, available at www.jneurosci.org as supplemental material). Because the number of total neurites was unaffected (supplemental Fig. S6A, available at www. jneurosci.org as supplemental material), the formation of multiple axons did not appear to be at the expense of dendrites. Thus, these data show that increased levels of AP180 and, to a lesser extent, CALM promote axogenesis.

AP180 and CALM deletion mutants do not localize to the axon or the dendrite To address the molecular basis for the functions of AP180 and CALM in developing neurons, we sought to examine the functional modules within the proteins. AP180 and CALM have similar overall structures, with an ANTH domain that binds to membrane lipids (Hao et al., 1997; Ford et al., 20001; Mao et al., 2001), and an unstructured C-terminal segment that contains short motifs for interacting with clathrin and AP2 (Lafer, 2002).

In our previous study, using CALM deletion mutants, we found that the ANTH domain was crucial for CALM on VAMP2 trafficking in non-neuronal cells (Harel et al., 2008). In this study, we likewise created HA-tagged AP180 constructs that either had the ANTH domain only (AP180ANTH-HA) or lacked it entirely (AP180-

\section{$\leftarrow$}

Figure 2. $\boldsymbol{A}-\boldsymbol{F}$, The effect of siRNA can be rescued by the expression of siRNA-resistant construct. $\boldsymbol{A}$, Immunolabeling of the axonal marker tau1 (red) in neurons coexpressing EGFP (green), the siRNA, and siRNA-resistant AP180 (AP180-RiR). Shown are merged confocal images. pSuper, the vector for the siRNA; Vector, the vector for AP180-RiR. Arrowheads indicate the axon. Scale bar, $10 \mu \mathrm{m}$. $\boldsymbol{B}$, Quantification of neurons with one axon. $n=45$ neurons in each group. Comparison is no rescue (AP180siRNA+Vector) versus rescue (AP180siRNA + AP180-RiR), ${ }^{* *} p<0.001$. C, The mean length of axons. $n=20-30$ neurons. Comparison is no rescue versus rescue, ${ }^{* *} p<0.001$. D, Immunolabeling of the dendritic marker MAP2 (red) in neurons coexpressing EGFP (green), the siRNA, and siRNA-resistant CALM (CALM-RiR). Arrowheads indicate dendrites. Scale bar, $10 \mu \mathrm{m}$. $\boldsymbol{E}$, Quantification of neurons containing MAP2-labeled dendrites. $n=45$ neurons in each group. Comparison is no rescue (CALMsiRNA+Vector) versus rescue (CALMsiRNA+CALM-L-RiR + CALM-S-RiR), ${ }^{* *} p<0.001$. $\boldsymbol{F}$, The total length of dendrites. $n=15-30$ neurons. Comparison is no rescue versus rescue, ${ }^{*} p<0.01$. Data represent means \pm SEM in $\boldsymbol{B}, \boldsymbol{C}, \boldsymbol{E}, \boldsymbol{F}$. 

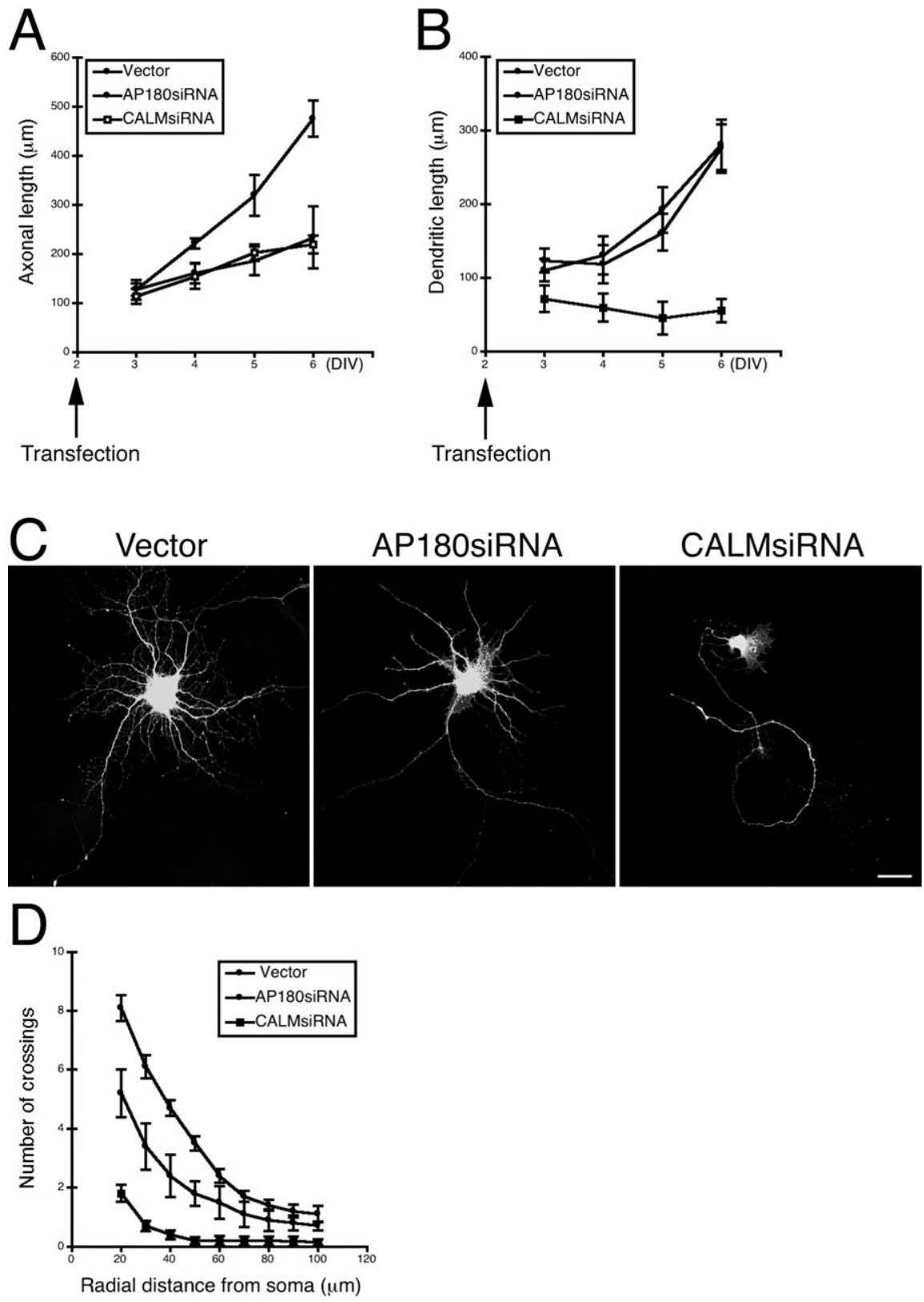

Figure 3. Effects of AP180siRNA or CALMsiRNA on axon and dendrite outgrowth in younger and older neurons. $\boldsymbol{A}, \boldsymbol{B}$, Quantification of axonal or dendritic length as a function of culture and transfection. $n=15-45$ neurons. Data represent means \pm SEM. DIV, days in vitro. C, Hippocampal neurons (10 div) expressing AP180siRNA or CALMsiRNA. Scale bar, $10 \mu \mathrm{m}$. Additional examples are shown in supplemental Figure $S 5 B$, available at www.jneurosci.org as supplemental material. $D$, Sholl analysis of the neurons shown in $C . n=20$ neurons in all groups. Data represent means \pm SEM.

ANTH $\Delta$-HA) (supplemental Fig. S7 $A, B$, available at www. jneurosci.org as supplemental material). We hypothesized that the ANTH domain would be required for AP180 and CALM on neurite outgrowth, and that the ANTH domain itself would function as a dominant-negative mutant. However, we discerned no morphological differences between those control neurons transfected with the parental vector only and those neurons transfected with the deletion mutants (Fig. 5A, $B$, left panels; data not shown).

To examine whether the AP180 and CALM deletion constructs were expressed and properly localized in the transfected neurons, we immunolabeled the HA-tagged constructs with an HA antibody. In both the neurons expressing wild-type AP180 and those expressing the wild-type CALM, we observed vivid immunofluorescence labeling in the soma and conspicuous la- beling in the neurites (Fig. $5 A, B$ ). This is similar in pattern to the expression of the endogenous AP180 and CALM (Yao et al., 2003). Surprisingly, in the neurons expressing AP180-ANTH or the AP180$\mathrm{ANTH} \Delta$, we detected HA labeling only in the soma, but not in any of the neurites (Fig. 5A, middle and bottom). Quantitative analysis, as determined by the ratio of immunofluorescent intensity in the neurites to that in the soma, revealed a markedly reduced labeling in the neurites (Fig. 5C). Notably, neurons expressing CALM deletion mutants also showed no HA immunolabeling in their neurites, and this was the case for both CALM-L (Fig. 5B,D) and CALM-S mutants (Fig. 5E) (data not shown). Our results indicate that the ANTH-only and the ANTH $\Delta$ mutants of AP180 and CALM failed to locate to neurites of developing hippocampal neurons. Therefore, in neurons, AP180 and CALM appear to require the specific sequences to reach their correct destinations, and these sequences are likely present in both the $\mathrm{N}$ and C-terminal parts of the proteins. Whether these sequences themselves serve as targeting signals or as determinants for the proper protein folding required for targeting remains to be determined.

\section{Electron microscopy reveals predicted and unexpected subcellular locations for AP180 and CALM}

The strikingly different phenotypes observed in neurons with reduced AP180 levels compared with those with reduced CALM levels suggest that the two assembly proteins do not perform the same function in the developing neuron. Assuming AP180 and CALM are required solely for the purpose of CCV assembly, the difference could reflect their association or involvement with distinct populations of CCVs. To address this question, we examined the subcellular distribution of AP180 and CALM by performing an immunogold electron microscopy analysis of neurons in the CA1 regions of the hippocampus in immature (P10) and mature (P37) rats. Notably, CCVs were frequently encountered in young hippocampal neurons; however, only a small portion of them had AP180 or CALM immunogold particles (Petralia and Yao, 2007; data not shown), indicating the transitory nature of CCVs and the even more transient association between the assembly proteins and CCVs. Nevertheless, if AP180 and CALM are exclusively associated with the CCVs at the plasma membrane, one would expect to see the immunogold particles located in close proximity to the cell surface. To obtain a quantitative assessment, we arbitrarily defined the region $\leq 100 \mathrm{~nm}$ (the diameter of a typical CCV) from the cell surface as the plasma membrane region (Fig. $6 \mathrm{Aa}, \mathrm{CCV}$ ) and the region $>100 \mathrm{~nm}$ from the surface as the intracellular region. We classified immunogold particles as plasma membrane associated 
when they were located within the plasma membrane region, and we applied this counting procedure to AP180 and CALM. We surveyed all micrographs ( 2000) that cover the entire CA1 stratum radiatum region ( 3 rats at each age), and we counted the profiles with a traceable plasma membrane and with $\geq 2$ gold particles within (Table 1). By this criterion, a large proportion of the AP180 and CALM labeling was in the plasma membrane region of neurons (Fig. 6Ab-d). However, in the case of both AP180 and CALM, there was also a substantial proportion of the labeling in the intracellular region (Fig. $6 A e-g)$. At a glance, we found that the percentages of AP180 ( 30\%) and CALM $(\sim 40 \%)$ in intracellular regions were comparable (Table 1). On closer examination, we also found that most of these intracellular AP180 particles were either poised at the edge of synaptic vesicle clusters or scattered sparsely in the cytoplasm of synaptic compartments (Fig. 6Ae,f). The intracellular CALM particles, however, frequently lay close to vesicular structures that resembled endosomes (Fig. $6 \mathrm{Ag})$.

To demonstrate CALM labeling in endosomes more distinctively, we performed additional immunogold labeling and examined the dendritic shaft and the soma, where endosomal structures are better unveiled. We examined both young (P10) (Fig. 6B) and adult (P37) (supplemental Fig. S8, available at www. jneurosci.org as supplemental material) neurons. We observed CALM labeling associated occasionally with CCV of the TGN (Fig. $6 B a$, arrowheads; supplemental Fig S $8 a, b$, available at www.jneurosci. org as supplemental material). Often, labeling was associated with early/sorting endosomes (Fig. $6 B b-d$, arrowheads; supplemental Fig. S8d,f, available at www. jneurosci.org as supplemental material); all of the gold particles in Figure $6 B b$ were associated with the tubular extensions of these endosomes. Also, labeling was commonly associated with tubulovesicular structures (Fig. 6Be, arrowheads; supplemental Fig. S8d, available at www. jneurosci.org as supplemental material). It is therefore likely that many, if not all, intracellular CALM associates with endosomes. Whether intracellular AP180 also associates with a particular type of internal organelles was not addressed here, because AP180 labeling in the dendritic shaft region was negligible.

Given what is currently known about secretory outposts in the development of dendrites (Horton et al., 2005; Hanus and

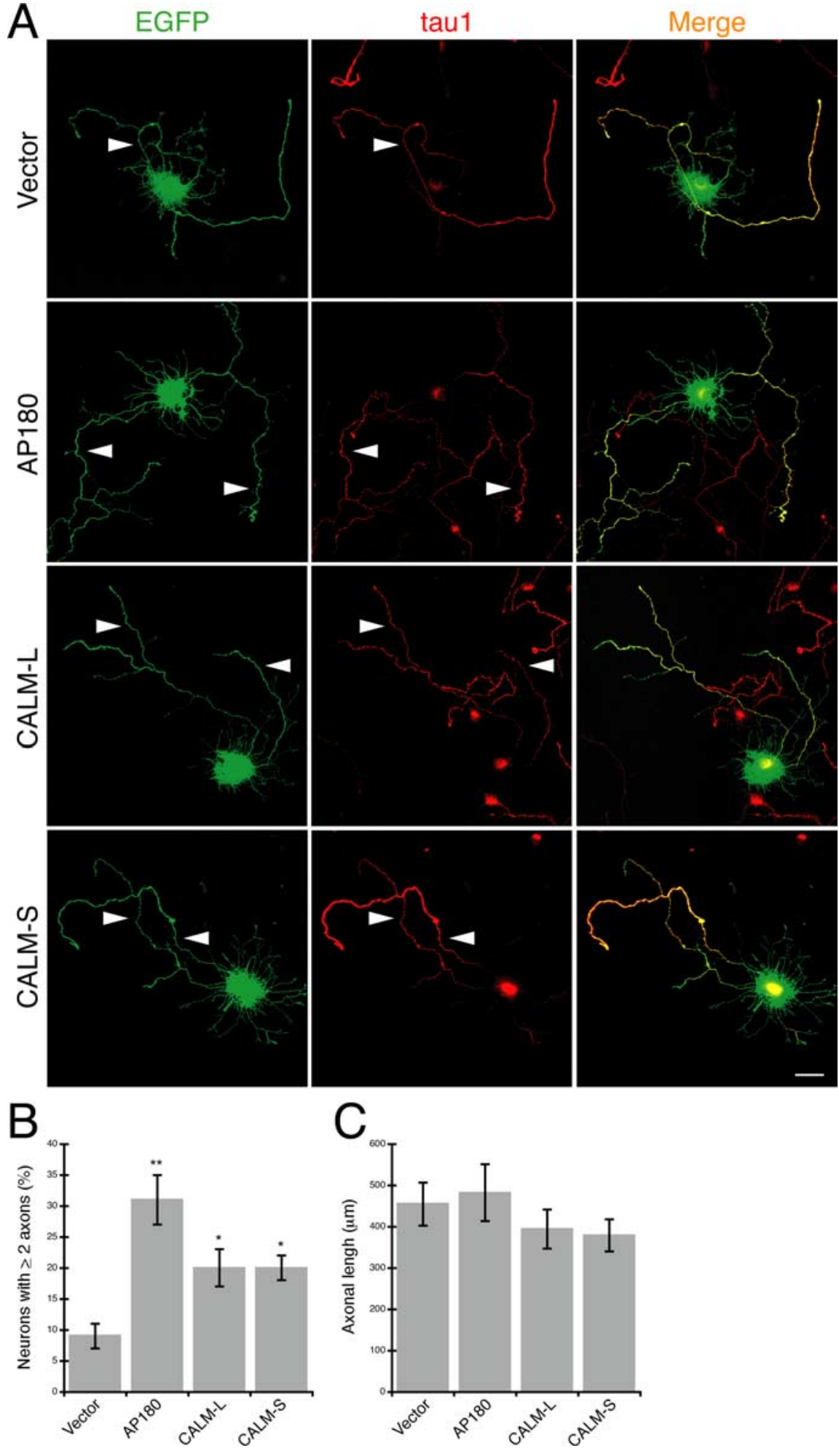

Figure 4. Overexpression of AP180 and CALM induces the hippocampal neurons to grow multiple axons. A, Immunolabeling of the axonal marker tau1 (red) in neurons coexpressing EGFP (green) and AP180 or CALM as indicated. Arrowheads indicate tau1-labeled axons. Scale $=10 \mu \mathrm{m}$. $\boldsymbol{B}$, Quantification of the neurons that have two or more tau1-labeled axons. ${ }^{*} p<0.01$; ${ }^{* *} p<0.001 ; n=45$ neurons. C, The mean length of the axons in neurons overexpressing AP180 or CALM. $n=45$ neurons. Data represent means $\pm \mathrm{SEM}$ in $\boldsymbol{B}, \boldsymbol{C}$.

Ehlers, 2008) and what we found about the role of CALM in dendrite outgrowth, we next examined the dendrite branch points. We frequently observed clusters of CALM immonogold particles directly associated with tubulovesicular structures that 


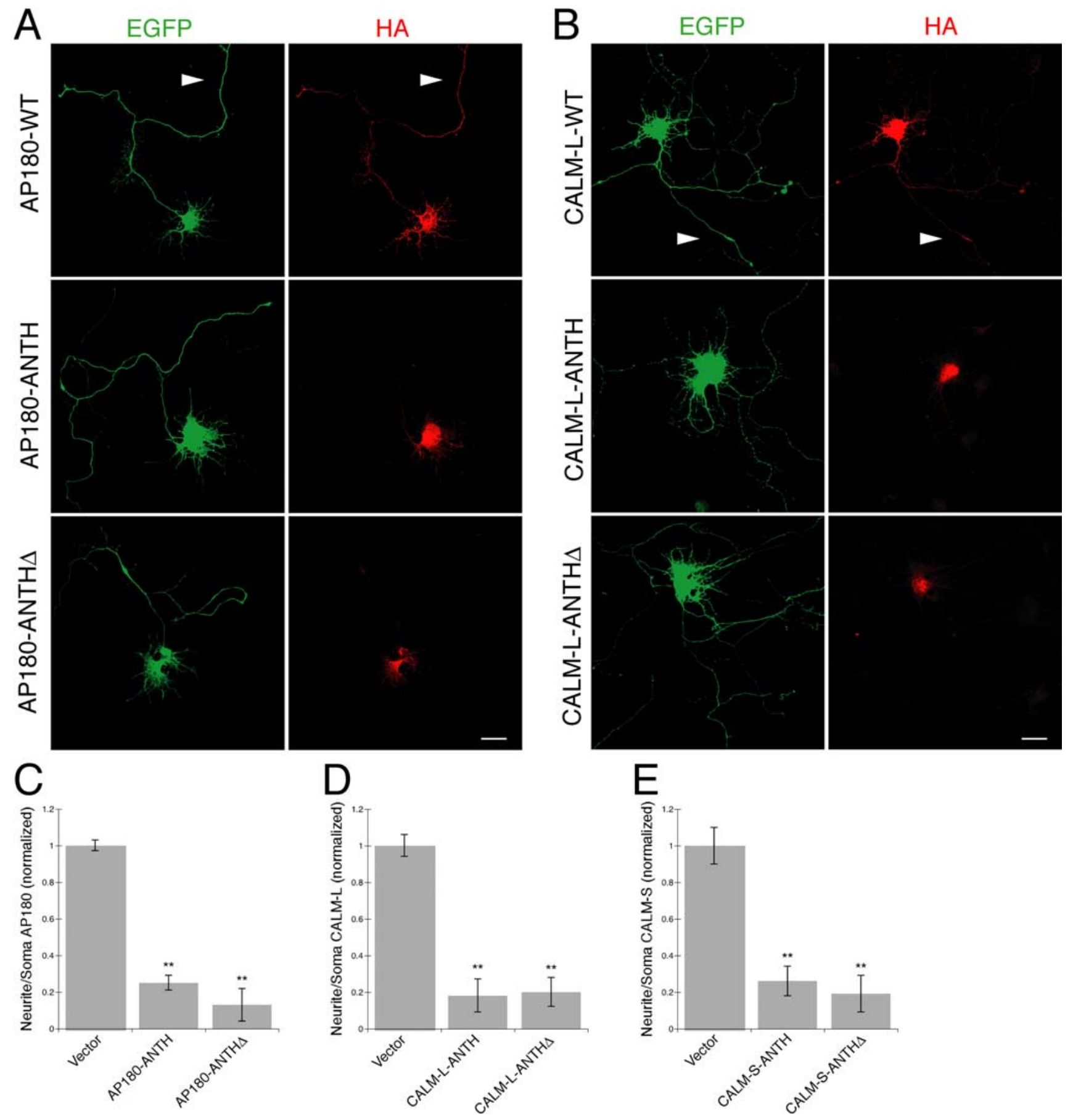

Figure 5. $A-E, A P 180$ and CALM deletion mutants fail to localize to neuronal processes. $A, B$, Immunolabeling of the HA (red) in the neurons coexpressing EGFP (green) and the HA-tagged AP180 $(\boldsymbol{A})$ or CALM-L $(\boldsymbol{B})$ as indicated. WT, Wild-type; ANTH, mutant with the ANTH domain only; ANTH $\Delta$, mutant with the ANTH domain deleted. Arrowheads indicate neurites bearing WT-HA labeling. Scale bars, $10 \mu \mathrm{m}$. C-E, Quantification of neuritic AP180-HA (C) or CALM-L-HA (D) or CALM-S-HA (E) in neurons expressing the indicated constructs. Data represent average intensity of AP180-HA (C) or CALM-HA $(\boldsymbol{D}, \boldsymbol{E})$ fluorescence in neurites divided by that in the soma. ${ }^{* *} p<0.001, n=30$ neurons in $\boldsymbol{C}, \boldsymbol{D} ; n=20$ neurons in $\boldsymbol{E}$.

are located immediately at or near the dendrite branch points (Fig. 7). It was unclear, however, whether these CALM-labeled and branch point-located tubulovesicular structures were Golgi outposts (Horton et al., 2005) because they did not exhibit the definitive classic structure of the Golgi stack.

\section{AP180 and CALM differentially participate in vesicular transport pathway}

It was reported previously that reducing CALM in non-neuronal cells appeared to disrupt the TGN and endosomal systems (Mey- erholz et al., 2005). A proteomic analysis, also of non-neuronal cells, revealed the abundance of CALM in AP1-containing CCVs, the CCVs budding from the TGN (Borner et al., 2006). Our ultrastructural study above demonstrated that, in neurons, a considerable fraction of CALM localizes to endosomal elements. Together, these observations indicate that, in addition to its canonical role at the plasma membrane (Tebar et al., 1999; Meyerholz et al., 2005), CALM is also involved in internal trafficking organelles.

To determine the consequences of AP180 and CALM reduction 

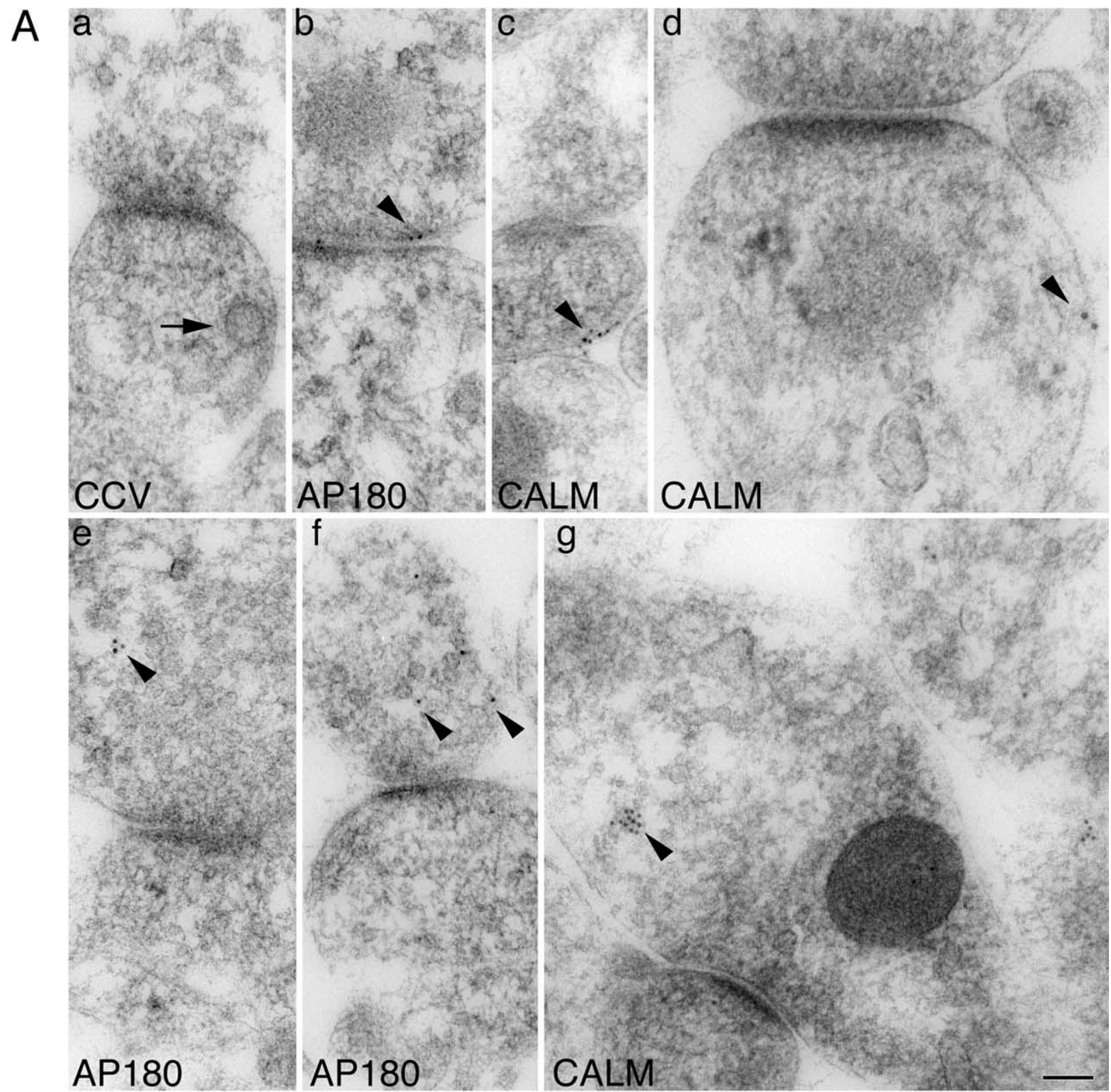

\section{AP180}
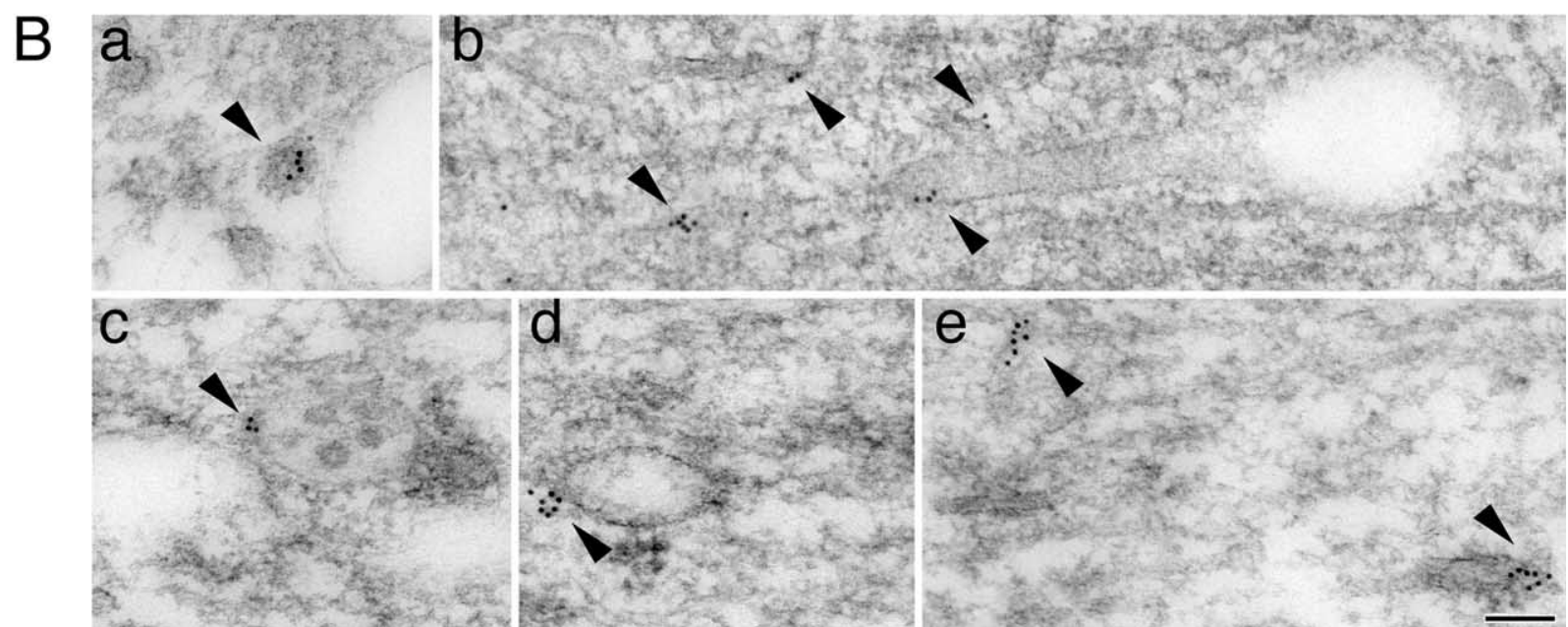

Figure 6. Subcellular localization of AP180 and CALM in the hippocampus. A, Immunogold labeling of AP180 or CALM in rat P10 hippocampal synapses. Arrow in Aa denotes a CCV beneath the plasma membrane. Arrowheads indicate the locations of AP180 or CALM immunogold particles. Ab, AP180 labeling at the plasma membrane; $\boldsymbol{A c}$, $\boldsymbol{A d}$, CALM labeling at the plasma membrane; $\boldsymbol{A e}$, $\boldsymbol{A f}$, intracellular AP180 labeling; $\boldsymbol{A g}$, intracellular CALM labeling. $\boldsymbol{B}$, Immunogold labeling of CALM in the P10 hippocampal soma or dendrites. Ba, CA1 stratum pyramidale cell body. Arrowhead marks CALM labeling on a CCV of the TGN. Bb, CA3 stratum lucidum large dendrite. $\boldsymbol{B} \boldsymbol{c}-\boldsymbol{B} \boldsymbol{e}$, Large dendrites in CA1 stratum pyramidale and stratum radiatum. Arrowheads in $\boldsymbol{B} \boldsymbol{b}-\boldsymbol{B} \boldsymbol{e}$ mark the $C A L M$ labeling on endosomes and their tubular extensions $(\boldsymbol{B b}-\boldsymbol{B d})$ or on unidentified tubular structures $(\boldsymbol{B e})$. Scale bars, $100 \mathrm{~nm}$. 

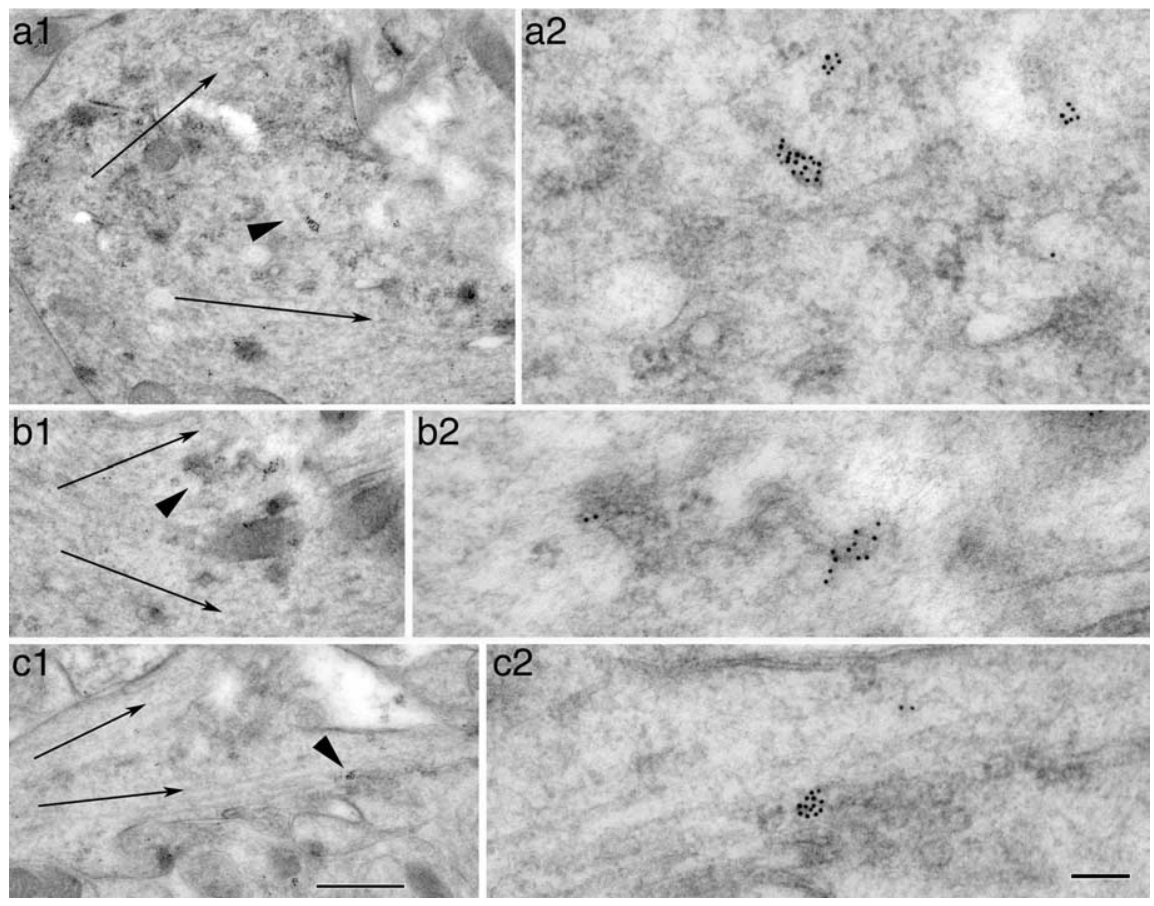

Figure 7. $\boldsymbol{a}-\boldsymbol{c}$, Immunogold labeling of CALM in tubulovesicular organelles at or near dendrite branch points in the CA1 stratum radiatum $(\boldsymbol{a}, \boldsymbol{b})$ and $\mathrm{CA} 1$ stratum oriens $(\boldsymbol{c})$ of the adult hippocampus. Thin arrows indicate dendrite branch orientations. Arrowheads in $\boldsymbol{a} \mathbf{1}, \boldsymbol{b 1}, \mathbf{c}$ indicate the area that is enlarged in the respective micrograph $\boldsymbol{a} \mathbf{2}, \boldsymbol{b} \mathbf{2}, \mathbf{c}$. Note that the CALM-labeled tubulovesicular organelles are located at the base of branch points separating the typical microtubule-rich dendrites. Scale bars: (in c1) for $\boldsymbol{a} 1-\boldsymbol{c 1}, 500 \mathrm{~nm}$; (in c2) for $\boldsymbol{a} 2-\boldsymbol{c} 2,100 \mathrm{~nm}$.

in vesicular transport, we examined intracellular trafficking organelles with a focus on their light microscopic appearances. We immunolabeled the neurons with specific markers for various subsets of internal organelles and examined the distribution and intensity of immunolabeling. We began by examining the cis-Golgi marker GM130. We found no difference in the GM130 labeling between neurons expressing either AP180siRNA or CALMsiRNA and the control neurons (supplemental Fig. S9A, available at www. jneurosci.org as supplemental material). Just as with the GM130 labeling, labeling for the TGN marker TGN38 did not distinguish between the siRNA-expressing neurons and the control neurons (supplemental Fig. S9B, available at www.jneurosci.org as supplemental material). Thus, neither AP180 nor CALM reduction elicits an effect on the localization and morphology of the cis-Golgi and TGN elements, at least not an effect discernible at the level of light microscopy. To examine the population of the AP1-containing CCVs, we labeled the neurons with an AP1-specific antibody. Despite the respective morphologies induced by AP180siRNA or CALMsiRNA, both types of neurons displayed the typical compact perinuclear labeling for AP1, and it was comparable in pattern and intensity to those observed in the control neurons (supplemental Fig. S9C, available at www.jneurosci.org as supplemental material). We also examined the additional markers including EEA1, Rab4, Rab11, and transferrin receptor. At the level of light microscopy, we did not observe any noticeable or consistent changes in these markers (data not shown).

Although knockdown of AP180 and CALM did not result in major changes of the organelle markers, we asked whether the reduction of AP180 or CALM nevertheless has physiological consequences on the trafficking organelles. To address this question, we examined the secretory transport. We used a temperaturesensitive mutant VSVG-GFP (Presley et al., 1997; Hirschberg et al., 2000). The VSVG-GFP mis-folds and is trapped in the ER at the nonpermissive temperature $\left(40^{\circ} \mathrm{C}\right)$. Upon shifting to a permissive temperature $\left(32^{\circ} \mathrm{C}\right)$, the protein exits ER, moves through the secretory pathway, and reaches the plasma membrane. The plasma membrane VSVG can be detected by labeling nonpermeabilized cells with an antibody specific to a luminal epitope of the protein (Lefrancios and Lyles, 1982). A measure of secretory transport therefore can be obtained by calculating the ratio of the mean intensity of surface VSVG immunoreactivity to the mean intensity of total VSVG-GFP.

We cotransfected AP180siRNA or CALMsiRNA with VSVG-GFP and incubated the neurons at $40^{\circ} \mathrm{C}$ for $1 \mathrm{~d}$. We did not observe any noticeable effects of the $40^{\circ} \mathrm{C}$ incubation on the viability or the morphology of the neurons (data not shown). We have demonstrated in an earlier experiment (Fig. $3 A, B$ ) that the siRNAs began to take effect by inducing the morphological phenotypes as soon as $1 \mathrm{~d}$ after transfection. Therefore, the $40^{\circ} \mathrm{C}-1 \mathrm{~d}$ paradigm was optimal for monitoring synchronously transported VSVG and determining the effects of the siRNAs in these neurons.

The total VSVG-GFP, revealed by the intensity of GFP, was similar between the AP180siRNA neurons or the CALMsiRNA neurons and the control neurons (Fig. $8 \mathrm{~A}$, left). The surface VSVG in the AP180siRNA neurons, revealed by immunolabeling using the luminal VSVG antibody, clearly and evenly outlined the neuronal soma and the proximal dendrites, in a pattern that was undistinguishable from that of the control neurons (Fig. 8A). The surface VSVG to total VSVG-GFP ratio was not different between the AP180siRNA neurons and the control neurons (Fig. 8 B), implying that the reduction of AP180 had no effect on secretory flux.

Unlike the AP180siRNA neurons, CALMsiRNA neurons exhibited a surface VSVG pattern that was notably different from that of the control neuron. On the majority of the neuronal surface, the labeling was scarcely visible (Fig. 8A; supplemental Fig. S10, available at www.jneurosci.org as supplemental material). On part of the surface, there was a propensity of slightly brighter labeling clusters, most of which were on protrusions (Fig. 8 A; supplemental Fig. S10, available at www.jneurosci.org as supplemental material). Quantitatively, the average intensity of the surface VSVG was reduced by $65 \%$ (Fig. $8 B$ ), indicating that the forward transport of VSVG had been disrupted in the CALMsiRNA neurons. These findings support the idea that CALM, but not AP180, likely participates in the secretory transport pathway in the neuron and, thus, that it may, at least in part, account for the divergent effects of AP180 and CALM on axons and dendrites.

\section{AP180- and CALM-mediated effects on neurite outgrowth are independent of polarized VAMP2 distribution}

In view of the well characterized role of AP180 and CALM in regulating $\mathrm{CCV}$ at the plasma membrane, we next examined endocytosis transport in the AP180- and CALM-deficient neurons. We focused on VAMP2, a component of synaptic vesicles (Taka- 
mori et al., 2006), and its distribution for two reasons. First, the asymmetrical distribution of VAMP2 in the neuron requires endocytosis (Grote and Kelly, 1996; Sampo et al., 2003). In the neuron, VAMP2 is delivered to the membrane surface of both the axon and dendrites but is removed selectively from the dendritic membrane (Sampo et al., 2003), a prerequisite for its enrichment in the axon and its targeting to synaptic vesicles. Second, the findings that AP180 and CALM were involved in VAMP2 endocytosis in invertebrates (Nonet et al., 1999; Bao et al., 2005; Dittman and Kaplan, 2006) and in mammalian non-neuronal cells (Harel et al., 2008) imply that, in mammalian neurons in which high-level sorting mechanisms are required, AP180 or CALM, either independently or together, can work to regulate endocytosis that directs the polarized distribution of proteins, including axonal VAMP2.

We used a VAMP2 construct that had been tagged with $\alpha$-bungarotoxin-binding sequence at its $\mathrm{C}$ terminus (VAMP2-BTX) (McCann et al., 2005; Harel et al., 2008). Given that the C terminus of VAMP2 positions on the extracellular side of the plasma membrane (Trimble, 1993), the surface VAMP2-BTX can be labeled in live neurons using fluorophore-conjugated BTX (Harel et al., 2008). To date, there is no available antibody specific to the $\mathrm{C}$ terminus of VAMP2 that would allow one to detect endogenous surface VAMP2; therefore, C-terminal-tagged VAMP2 constructs (this study; Sampo et al., 2003) serve as useful tools to study surface VAMP2 and its trafficking.

We began by transfecting cultured hippocampal neurons with the VAMP2-BTX and labeled surface VAMP2-BTX with Alexa555-BTX at $4^{\circ} \mathrm{C}$. The axon was brightly labeled and the labeling was more intense in its distal segment; the dendrites and soma, however, were not labeled (supplemental Fig. S11A, available at www. jneurosci.org as supplemental material). This polarized VAMP2 labeling pattern is similar to the pattern of hippocampal neurons expressing a different C-terminal-tagged VAMP2 (VAMP2-CFP; Sampo et al., 2003).

We next cotransfected neurons with the VAMP2-BTX, AP180siRNA, or CALMsiRNA, and EGFP, followed by labeling surface VAMP2-BTX. The control neurons exhibited the typical polarized VAMP2 pattern with detectable labeling only on the axon, none on the dendrites (Fig. 9A). In contrast, in the AP180siRNA neurons, surface VAMP2-BTX was not limited to the axon; rather, the labeling became readily detectable on all neurites and was equally intense in the axon and the dendrites (Fig. 9A). Additional examples of the neurons at different developmental stages in culture showed that the effect of AP180siRNA on the loss of polarized distribution of VAMP2 was similar, re-

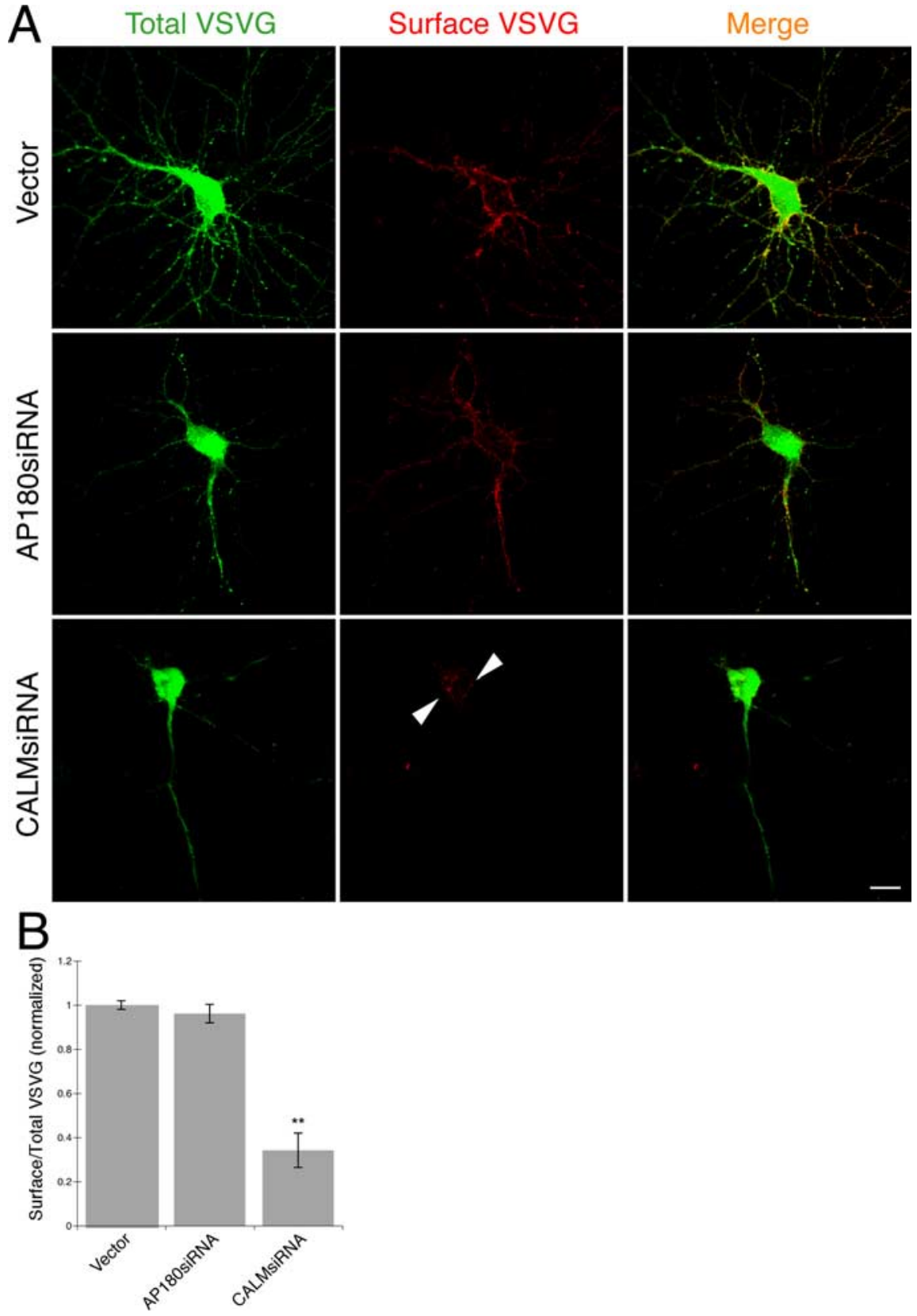

Figure 8. Knockdown of CALM but not AP180 disrupts forward transport. $\boldsymbol{A}$, Confocal images showing total VSVG-GFP (green) and surface VSVG (red) in the control and AP180siRNA neurons (16 div) or CALMsiRNA neurons (16 div). Arrowheads indicate surface VSVG clusters. Scale bar, $10 \mu \mathrm{m}$. $\boldsymbol{B}$, Quantification of surface to total ratio of VSVG in the control and the siRNA neurons. ${ }^{* *} p<0.001, n=20$ neurons in all groups.

gardless of the maturity of the neurons (supplemental Fig. S11B, available at www.jneurosci.org as supplemental material). To address this issue more quantitatively, we measured and computed the ratio of axon to dendrite surface VAMP2-BTX fluorescence; the value in the AP180siRNA neurons was significantly lower than the value in the control neurons (Fig. 9B).

In the case of the CALMsiRNA-transfected neurons, we categorized them into two populations based on their morphological phenotype: the prominent phenotype, with one axon and no dendrite, and the seemingly unchanged phenotype (Fig. 9A). In the population of the neurons with the typical CALMsiRNAinduced phenotype, surface VAMP2-BTX labeling was bright and clear on the axon, the only remaining neurite in many cases, and on the soma (Fig. 9A, bottom). In the population of the 


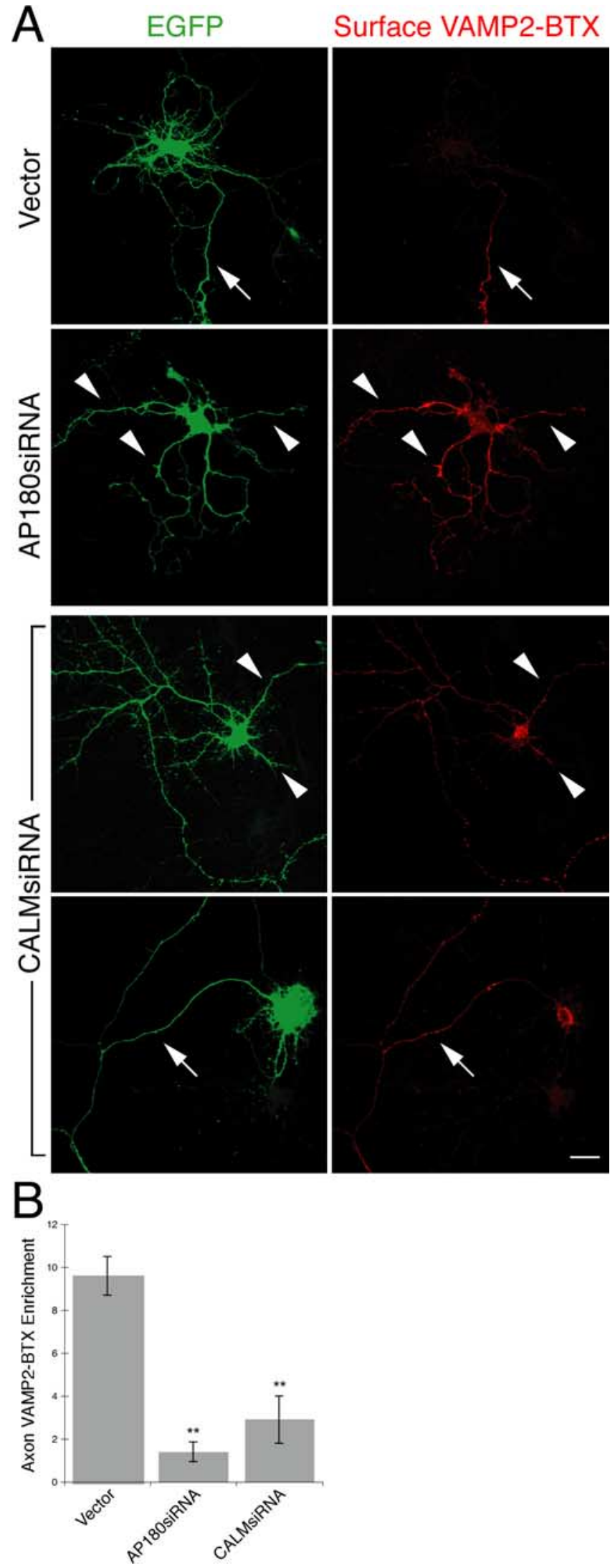

Figure 9. Knockdown of AP180 and CALM disturbs VAMP2 distribution. Live neurons coexpressing EGFP (green), VAMP2-BTX, and the siRNA were labeled with Alexa555-BTX (red) at $4{ }^{\circ} \mathrm{C}$. The neurons were then fixed and confocal images were acquired. $A$, Surface VAMP2-BTX in the AP180siRNA neurons or the CALMsiRNA neurons. In the control neuron, surface VAMP2-BTX is detected only on the axon (arrow). In the AP180siRNA neuron, surface VAMP2 becomes detectable on all neurites (arrowheads). Among the CALMsiRNA neurons, some of them have a seem- neurons with multiple neurites, we found that surface VAMP2BTX was present in all neurites, axons, and dendrites (Fig. 9A, top). Quantitative analysis of this population showed a loss of the polarized distribution of VAMP2 similar to that seen in the AP180siRNA neurons (Fig. 9B).

To determine whether the knockdown of AP180 or CALM affected the expression of total VAMP2, we immunolabeled the respective neurons with a VAMP2-specific antibody. The antibody labeled both endogenous VAMP2 and ectopic VAMP2BTX. Neither AP180siRNA neurons nor CALMsiRNA neurons displayed detectable changes in the labeling pattern or intensity of total VAMP2 (data not shown).

Therefore, although AP180 and CALM seem to be involved, both individually and in combination, in vesicular transport, we demonstrate that, in the case of VAMP2 in the hippocampal neuron, both proteins regulate VAMP2 endocytosis, a mechanism necessary for its targeting to the axon.

Did VAMP2 missorting then contribute to the AP180siRNAor CALMsiRNA-induced growth defect in hippocampal neurons that we have observed in this study? If so, we reasoned that disrupting VAMP2 endocytosis should produce the same, or similar, phenotypes in neuron growth. To test this hypothesis, we overexpressed the neurons with the VAMP2M46A-BTX, a mutant defective in endocytosis (Grote and Kelly, 1996). As expected, the mutant VAMP2M46A failed to undergo endocytosis from the dendritic membrane of the VAMP2M46A-expressing neurons, leading to intense surface BTX labeling on all neurites (Fig. 10). However, we observed no morphological change when comparing neurons expressing the mutant VAMP2M46A with neurons expressing the wild-type VAMP2 (Fig. 10, left). Moreover, despite the fact that the VAMP2M46A-expressing neurons exhibited VAMP2 missorting to the dendritic surface (Fig. 10), a pattern strikingly similar to that seen in the AP180siRNA neurons and CALMsiRNA neurons (Fig. 9A), the mutant VAMP2M46A did not result in any morphology resembling either the AP180siRNA- or the CALMsiRNA-induced changes. Thus, our results suggest that AP180- and CALM-mediated effects on axon and dendrite growth are independent of the polarized distribution of VAMP2. Whether the defect in VAMP2 distribution causes other functional changes in the neuron is a further question not addressed here.

\section{Discussion}

In this study, we use cultured embryonic hippocampal neurons as a model system to study the roles that clathrin assembly proteins AP180 and CALM play in regulating the outgrowth of axons and dendrites. We provide evidence that AP180 and CALM are each important for the establishment of the neuritic architecture and that each protein is devoted to specific aspects of the growth process. Selective depletion of AP180 impairs axogenesis, in many cases resulting in the inability of the neuron to establish polarity, whereas overexpression of AP180 induces many neurons to form multiple axons. Reduction of CALM levels does not affect axogenesis, but it does severely impair the growth of dendrites, resulting in neurons with only an axon.

ingly unaltered morphology (top), but they express surface VAMP2 on all neurites (arrowheads); others have most of their dendrites missing (bottom), and surface VAMP2 is brightly labeled on the axon. Scale bar, $10 \mu \mathrm{m}$. B, Quantification of VAMP2 axon enrichment. Data represent average intensity of $B T X$ fluorescence in the longest neurite divided by that in all other neurites. ${ }^{* *} p<0.001, n=30$ neurons for the control and AP180siRNA; $n=15$ neurons for CALMsiRNA. 
Evidence that links intracellular trafficking and cell polarity has been mostly derived from studies of epithelial cells (Fölsch, 2008). For example, Drosophila mutants with defective endocytosis lose polarity in their epithelial cells ( $\mathrm{Lu}$ and Bilder, 2005); disruption of Rab8 GTPasemediated trafficking results in the mislocation of apical proteins in intestinal epithelial cells (Sato et al., 2007); and disturbance of clathrin-mediated trafficking in MadinDarby canine kidney epithelial cells selectively impairs the distribution of basolateral proteins (Deborde et al., 2008). Recent studies of neurons have shown that a functional secretory route is required for dendritic growth (Horton et al., 2005; Ye et al., 2007), emphasizing the role that intracellular trafficking plays in the growth of the neuron, a cell type having a morphology and membrane specialization that are much more complex than those of epithelial cells.

The present study provides evidence for the critical involvement of two novel proteins, AP180 and CALM, in the establish-

ment and quantitative regulation of neuronal polarity in developing neurons. Both AP180 and CALM have previously been shown to play roles in clathrin assembly, and our findings of distinctive phenotypes induced by the knockdown of AP180 and CALM suggest that these two clathrin assembly proteins likely associate with different subsets of CCVs or trafficking organelles in embryonic neurons. Judging by their anatomical location at the subcellular level (Figs. 6, 7; supplemental Fig. S8, available at www.jneurosci.org as supplemental material), CALM, but not AP180, affiliates with internal trafficking organelles, suggesting that CALM is involved in the transport pathway in addition to endocytosis at the plasma membrane. This finding confirms and extends previous observations of CALM in AP1-containing CCVs (Borner et al., 2006) and of disrupted TGN and endosomal system in CALM-depleted non-neuronal cells (Meyerholz et al., 2005).

Having described the massing of CALM at intracellular organelles, we turn to investigate the specific transport pathway in which CALM carries out its function. The striking parallels between the phenotype induced by CALM knockdown in our study and the phenotype induced by secretory transport disruption in other studies (Horton et al., 2005) suggest a functional link between CALM and the forward secretory pathway. When siRNA was used to reduce CALM levels, we found severely suppressed dendritic growth, a phenotype similar in degree and appearance to what has been observed in neurons with disrupted post-Golgi secretory trafficking (Horton et al., 2005). Our demonstration of markedly reduced surface VSVG levels in the CALM-deficient neurons (Fig. 8) highlights the importance of the secretory pathway in the growth of the dendrite and suggests that, in the neuron, the secretory pathway relies on CALM.

Regarding the roles of AP180 and CALM in endocytosis, genetic, biochemical, and anatomical studies using various experimental models have established that both AP180 and CALM promote the assembly of CCVs derived from the plasma membrane during endocytosis. In developing neurons, the close proximity of a large proportion of AP180 and CALM immunogold particles to the plasma membrane (Fig. 6A) is consistent with their established role in endocytosis. Do AP180 and CALM play a role that is
Surface VAMP2-BTX
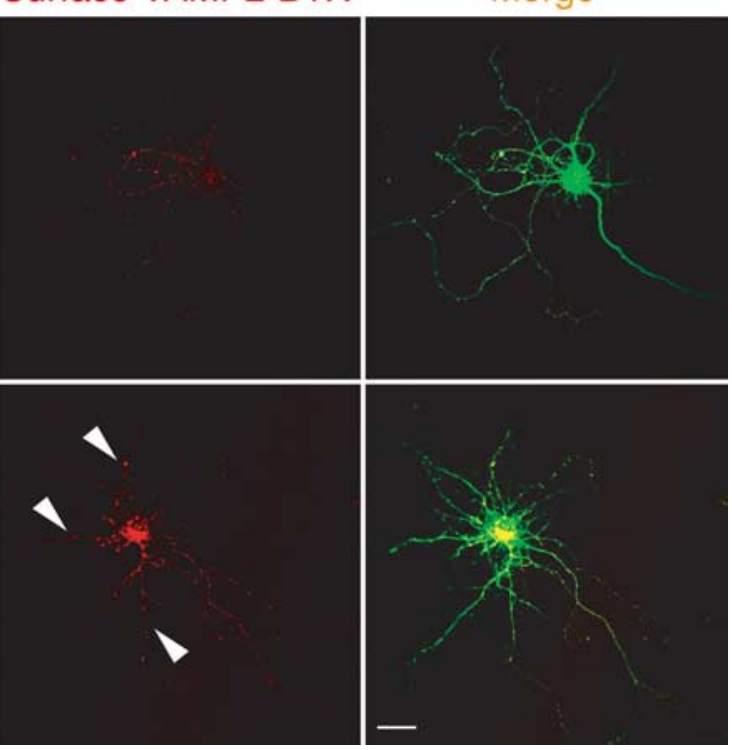

Figure 10. Defective VAMP2 endocytosis does not affect neuron morphology. Live neurons coexpressing EGFP (green) and wild-type VAMP2-BTX or mutant VAMP2M46A-BTX were labeled with Alexa555-BTX (red) at $4^{\circ} \mathrm{C}$ and then fixed. Although the mutant VAMPM46A-BTX is mislocated to all neurites (arrowheads), the morphology of the neuron is not changed. Scale bar, $10 \mu \mathrm{m}$.

merely redundant? Or does each protein execute specialized controls over CCVs during endocytosis? One possibility is that CCV formation requires fast and efficient temporal regulation and that this regulation is ensured by the presence of both AP180 and CALM. Another possibility is that the proteins provide additional and much needed spatial regulation to endocytosis in specific compartments (e.g., axons and dendrites) of the neuron.

It is also likely that different assembly proteins direct different cargos in the process of CCV assembly; therefore, the absence of either protein would result in failed endocytosis of a different repertoire of cargos that could elicit diverse consequences in the neuron. Whether this is true for AP180 and CALM, and if so, which specific cargos are regulated by either protein will require further investigation. All data thus far have shown that one cargo, VAMP2, appears to be directed by both AP180 and CALM. We confirmed that in the mammalian neuron the knockdown of either AP180 and CALM similarly impairs the endocytosis of VAMP2 from the dendritic surface. Although VAMP2 distribution does not seem to have a significant role in the overall morphology of young neurons, we find for the first time that AP180and CALM-mediated intracellular trafficking is important for the proper development of the neuron.

\section{References}

Ahle S, Ungewickell E (1986) Purification and properties of a new clathrin assembly protein. EMBO J 5:3143-3149.

Bao H, Daniels RW, MacLeod GT, Charlton MP, Atwood HL, Zhang B (2005) AP180 maintains the distribution of synaptic and vesicle proteins in the nerve terminal and indirectly regulates the efficacy of Ca2+triggered exocytosis. J Neurophysiol 94:1888-1903.

Borner GH, Harbour M, Hester S, Lilley KS, Robinson MS (2006) Comparative proteomics of clathrin-coated vesicles. J Cell Biol 175:571-578.

Brodsky FM, Chen CY, Knuehl C, Towler MC, Wakeham DE (2001) Biological basket weaving: formation and function of clathrin-coated vesicles. Annu Rev Cell Dev Biol 17:517-568.

Brummelkamp TR, Bernards R, Agami R (2002) A system for stable expression of short interfering RNAs in mammalian cells. Science 296:550-553.

Deborde S, Perret E, Gravotta D, Deora A, Salvarezza S, Schreiner R, Rodriguez-Boulan E (2008) Clathrin is a key regulator of basolateral polarity. Nature 452:719-723. 
Dittman JS, Kaplan JM (2006) Factors regulating the abundance and localization of synaptobrevin in the plasma membrane. Proc Natl Acad Sci U S A 103:11399-11404.

Dreyling MH, Martinez-Climent JA, Zheng M, Mao J, Rowley JD, Bohlander SK (1996) The $t(10 ; 11)(p 13 ; q 14)$ in the U937 cell line results in the fusion of the AF10 gene and CALM, encoding a new member of the AP-3 clathrin assembly protein family. Proc Natl Acad Sci USA 93:4804-4809.

Fölsch H (2008) Regulation of membrane trafficking in polarized epithelial cells. Curr Opin Cell Biol 20:208-213.

Ford MG, Pearse BM, Higgins MK, Vallis Y, Owen DJ, Gibson A, Hopkins CR, Evans PR, McMahon HT (2001) Simultaneous binding of PtdIns(4,5)P2 and clathrin by AP180 in the nucleation of clathrin lattices on membranes. Science 291:1051-1055.

Garrido JJ, Fernandes F, Giraud P, Mouret I, Pasqualini E, Fache MP, Jullien F, Dargent B (2001) Identification of an axonal determinant in the C-terminus of the sodium channel $\mathrm{Na}(\mathrm{v})$ 1.2. EMBO J 20:5950-5961.

Goldstein B, Macara IG (2007) The PAR proteins: fundamental players in animal cell polarization. Dev Cell 13:609-622.

Grote E, Kelly RB (1996) Endocytosis of VAMP is facilitated by a synaptic vesicle targeting signal. J Cell Biol 132:537-547.

Hanus C, Ehlers MD (2008) Secretory outposts for the local processing of membrane cargo in neuronal dendrites. Traffic 9:1437-1445.

Hao W, Tan Z, Prasad K, Reddy KK, Chen J, Prestwich GD, Falck JR, Shears SB, Lafer EM (1997) Regulation of AP-3 function by inositides. Identification of phosphatidylinositol 3,4,5-trisphosphate as a potent ligand. J Biol Chem 272:6393-6398.

Harel A, Wu F, Mattson MP, Morris CM, Yao PJ (2008) Evidence for CALM in directing VAMP2 trafficking. Traffic 9:417-429.

Hirschberg K, Phair RD, Lippincott-Schwartz J (2000) Kinetic analysis of intracellular trafficking in single living cells with vesicular stomatitis virus protein G-green fluorescent protein hybrids. Methods Enzymol 327:69-89.

Horton AC, Rácz B, Monson EE, Lin AL, Weinberg RJ, Ehlers MD (2005) Polarized secretory trafficking directs cargo for asymmetric dendrite growth and morphogenesis. Neuron 48:757-771.

Jareb M, Banker G (1997) Inhibition of axonal growth by brefeldin A in hippocampal neurons in culture. J Neurosci 17:8955-8963.

Kaech S, Banker G (2006) Culturing hippocampal neurons. Nat Protoc 1:2406-2415.

Kohtz DS, Puszkin S (1988) A neuronal protein (NP185) associated with clathrin-coated vesicles. Characterization of NP185 with monoclonal antibodies. J Biol Chem 263:7418-7425.

Lafer EM (2002) Clathrin-protein interactions. Traffic 3:513-520.

Lefrancios L, Lyles DS (1982) The interactionof antiody with the major surface glycoprotein of vesicular stomatitis virus. I. Analysis of neutralizing epitopes with monoclonal antibodies. Virology 121:157-167.

Lu H, Bilder D (2005) Endocytic control of epithelial polarity and proliferation in Drosophila. Nat Cell Biol 7:1232-1239.

Mao Y, Chen J, Maynard JA, Zhang B, Quiocho FA (2001) A novel all helix fold of the AP180 amino-terminal domain for phosphoinositide binding and clathrin assembly in synaptic vesicle endocytosis. Cell 104:433-440.

Marco E, Wedlich-Soldner R, Li R, Altschuler SJ, Wu LF (2007) Endocytosis optimizes the dynamic localization of membrane proteins that regulate cortical polarity. Cell 129:411-422.

McCann CM, Bareyre FM, Lichtman JW, Sanes JR (2005) Peptide tags for labeling membrane proteins in live cells with multiple fluorophores. Biotechniques 38:945-952.

Meyerholz A, Hinrichsen L, Groos S, Esk PC, Brandes G, Ungewickell EJ (2005) Effect of clathrin assembly lymphoid myeloid leukemia protein depletion on clathrin coat formation. Traffic 6:1225-1234.

Morgan JR, Zhao X, Womack M, Prasad K, Augustine GJ, Lafer EM (1999) A role for the clathrin assembly domain of AP180 in synaptic vesicle endocytosis. J Neurosci 19:10201-10212.

Morris SA, Schröder S, Plessmann U, Weber K, Ungewickell E (1993) Clathrin assembly protein AP180: primary structure, domain organization and identification of a clathrin binding site. EMBO J 12:667-675.

Murphy JE, Pleasure IT, Puszkin S, Prasad K, Keen JH (1991) Clathrin as- sembly protein AP-3. The identity of the $155 \mathrm{~K}$ protein, AP 180 , and NP185 and demonstration of a clathrin binding domain. J Biol Chem 266:4401-4408.

Nonet ML, Holgado AM, Brewer F, Serpe CJ, Norbeck BA, Holleran J, Wei L, Hartwieg E, Jorgensen EM, Alfonso A (1999) UNC-11, a Caenorhabditis elegans AP180 homologue, regulates the size and protein composition of synaptic vesicles. Mol Biol Cell 10:2343-2360.

Owen DJ, Collins BM, Evans PR (2004) Adaptors for clathrin coats: structure and function. Annu Rev Cell Dev Biol 20:153-191.

Petralia RS, Wenthold RJ (1999) Immunocytochemistry of NMDA receptors. In: Methods in molecular biology: NMDA receptor protocols (Li M, ed), pp 73-92. Totowa, NJ: Humana.

Petralia RS, Yao PJ (2007) AP180 and CALM in the developing hippocampus: expression at the nascent synapse and localization to trafficking organelles. J Comp Neurol 504:314-327.

Petralia RS, Sans N, Wang YX, Wenthold RJ (2005) Ontogeny of postsynaptic density proteins at glutamatergic synapses. Mol Cell Neurosci 29:436-452.

Presley JF, Cole NB, Schroer TA, Hirschberg K, Zaal KJ, Lippincott-Schwartz J (1997) ER-to-Golgi transport visualized in living cells. Nature 389:81-85

Robinson MS (2004) Adaptable adaptors for coated vesicles. Trends Cell Biol 14:167-174

Sampo B, Kaech S, Kunz S, Banker G (2003) Two distinct mechanisms target membrane proteins to the axonal surface. Neuron 37:611-624.

Sato T, Mushiake S, Kato Y, Sato K, Sato M, Takeda N, Ozono K, Miki K, Kubo Y, Tsuji A, Harada R, Harada A (2007) The Rab8 GTPase regulates apical protein localization in intestinal cells. Nature 448:366-369.

Shi SH, Jan LY, Jan YN (2003) Hippocampal neuronal polarity specified by spatially localized mPar3/mPar6 and PI 3-kinase activity. Cell 112:63-75.

Sousa R, Tannery NH, Zhou S, Lafer EM (1992) Characterization of a novel synapse-specific protein. I. Developmental expression and cellular localization of the F1-20 protein and mRNA. J Neurosci 12:2130-2143.

Sternberger LA, Harwell LW, Sternberger NH (1982) Neurotypy: regional individuality in rat brain detected by immunocytochemistry with monoclonal antibodies. Proc Natl Acad Sci U S A 79:1326-1330.

Takamori S, Holt M, Stenius K, Lemke EA, Grønborg M, Riedel D, Urlaub H, Schenck S, Brügger B, Ringler P, Müller SA, Rammner B, Gräter F, Hub JS, De Groot BL, Mieskes G, Moriyama Y, Klingauf J, Grubmüller H, Heuser J, et al. (2006) Molecular anatomy of a trafficking organelle. Cell 127:831-846.

Tebar F, Bohlander SK, Sorkin A (1999) Clathrin assembly lymphoid myeloid leukemia (CALM) protein: localization in endocytic-coated pits, interactions with clathrin, and the impact of overexpression on clathrinmediated traffic. Mol Biol Cell 10:2687-2702.

Trimble WS (1993) Analysis of the structure and expression of the VAMP family of synaptic vesicle proteins. J Physiol Paris 87:107-115.

Yao PJ, Coleman PD, Calkins DJ (2002) High-resolution localization of clathrin assembly protein AP180 in the presynaptic terminals of mammalian neurons. J Comp Neurol 447:152-162.

Yao PJ, Zhang P, Mattson MP, Furukawa K (2003) Heterogeneity of endocytic proteins: distribution of clathrin adaptor proteins in neurons and glia. Neuroscience 121:25-37.

Yao PJ, Petralia RS, Bushlin I, Wang Y, Furukawa K (2005) Synaptic distribution of the endocytic accessory proteins AP180 and CALM. J Comp Neurol 481:58-69.

Ye B, Zhang Y, Song W, Younger SH, Jan LY, Jan YN (2007) Growing dendrites and axons differ in their reliance on the secretory pathway. Cell 130:717-729.

Ye W, Lafer EM (1995) Bacterially expressed F1-20/AP-3 assembles clathrin into cages with a narrow size distribution: implications for the regulation of quantal size during neurotransmission. J Neurosci Res 41:15-26.

Zhang B, Koh YH, Beckstead RB, Budnik V, Ganetzky B, Bellen HJ (1998) Synaptic vesicle size and number are regulated by a clathrin adaptor protein required for endocytosis. Neuron 21:1465-1475.

Zhou S, Tannery NH, Yang J, Puszkin S, Lafer EM (1993) The synapsespecific phosphoprotein F1-20 is identical to the clathrin assembly protein AP-3. J Biol Chem 268:12655-12662. 